\title{
Experience-dependent Plasticity in the Inferior Colliculus: A Site for Visual Calibration of the Neural Representation of Auditory Space in the Barn Owl
}

\author{
Michael S. Brainard and Eric I. Knudsen \\ Department of Neurobiology, Stanford University, Stanford, California 94305-5401
}

The optic tectum (homolog of the superior colliculus) contains mutually allgned neural maps of auditory and visual space. During development, the organization of the auditory map is guided by spatial information provided by vision: barn owls raised wearing prismatic spectacles, which optically shift the visual field and the visual map in the optic tectum, develop an auditory map that is shifted by an approximately equivalent amount, such that alignment between the two maps is preserved (Knudsen and Brainard, 1991). In this study we investigated whether this shift in the auditory map is intrinsic to the optic tectum or whether it reflects plasticity at an earlier stage in the auditory pathway.

Owls were raised wearing prismatic spectacles that displaced the visual field by $23^{\circ}$ to the left or right. This manipulation alters the normal correspondence between locations in the visual field and interaural time difference (ITD), the primary cue for the azimuth of a sound source. In normal owls and in owls with at least $150 \mathrm{~d}$ of prism experience, extracellular unit recordings were used to assess the representations of ITD at anatomically and physiologically defined sites in the optic tectum and in the two prior stages of the auditory pathway, the external and central nuclei of the inferior colliculus (ICX and ICC).

In the optic tectum of normal owls, the values of ITD to which units responded most strongly (best ITDs) varied systematically with the azimuths of unit visual receptive fields (VRFs). In the prism-reared owls, best ITDs were shifted from normal toward the values of ITD produced by sounds at the locations of the units' optically displaced VRFs.

In the ICx of prism-reared owls, the representation of ITD also was shifted from normal, by an amount and in a direction that could completely account for the shift in ITD measured in the optic tectum. At some sites in the ICX, the shift in ITD tuning was apparent within the first $7-8 \mathrm{msec}$ of the response; shifted tuning at such short latencies argues that the altered representation of ITD in the ICx reflects plasticity in the ascending auditory pathway, and is not the result of descending activity from higher auditory centers.

In the ICc, which immediately precedes the ICX in the as-

Received Jan. 8, 1993; revised Apr. 28, 1993; accepted May 6, 1993.

We thank Catherine Carr, Dan Feldman, Joachim Mogdans, William Newsome and Carla Shatz for reviewing the manuscript. This work was supported by NIH R01 DC 00155-14 and T32 NS 07158-13.

Correspondence should be addressed to Michael S. Brainard, Department of Neurobiology, Fairchild Science Building, Stanford University School of Medicine, Stanford, CA 94305-5401.

Copyright (C) 1993 Society for Neuroscience $0270-6474 / 93 / 134589-20 \$ 05.00 / 0$ cending pathway, the representation of ITD was normal. The results indicate that the visual instruction of auditory spatial tuning of neurons in the optic tectum reflects plasticity at the level of the $\mathrm{ICx}$, the site where the auditory map of space is first synthesized.

[Key words: Tyto alba, sound localization, superior colliculus, optic tectum, auditory plasticity, auditory development, sensory experience, neural maps]

The nervous system infers the location of a sound source from timing, intensity, and spectral cues available in the signals at the two ears. Accurate sound localization requires correct associations of these cues with the sound source locations that produce them. Spatial information about sound source location provided by vision can have a powerful influence on the formation of these associations. For example, in animals reared without vision, sound localization accuracy can be degraded (humans: Fisher, 1964; Jones, 1975; Wanet and Veraart, 1985; rats: Spiegelman and Bryden, 1967; owls: Knudsen et al., 1991). Furthermore, in animals chronically exposed to an optically displaced visual field, sound localization may be adjusted to match the visual displacement (humans: Lackner, 1973; owls: Knudsen and Knudsen, 1989).

A prior study demonstrated that a neural correlate of the visual instruction of sound localization is found in the barn owl's optic tectum. Neurons in the optic tectum (homolog of the mammalian supcrior colliculus) have spatially restricted receptive fields for visual and auditory stimuli (Knudsen, 1982). At a given site in the optic tectum, visual and auditory receptive fields are aligned, and the locations of receptive fields vary systematically across the optic tectum to form mutually aligned maps of visual and auditory space. When owls are raised wearing prismatic glasses, which optically displace the visual field and create a mismatch between the visual and auditory maps in the optic tectum, the auditory map shifts in the optic tectum, restoring alignment between the two maps (Knudsen and Brainard, 1991).

In contrast to the visual map in the optic tectum, which results from a direct topographic projection from the retina, the auditory map is synthesized from sound localization cues in a series of stages. In principle, the shift in the auditory map observed at the level of the optic tectum could be due to plasticity at any of these earlier stages. In this study, we describe the visually induced changes in the tuning of tectal units for sound localization cues and we demonstrate where in the auditory pathway these visually guided changes occur.

In barn owls, the tuning of neurons in azimuth and elevation 
is due primarily to their tuning for the relative timing and sound pressure level (or intensity), respectively, of the signals at the two ears (Moiseff and Konishi, 1981a; Olsen et al., 1989). Parallel neural pathways extract information about interaural timing differences (ITD) and interaural level differences (ILD) (for a review of these pathways, see Konishi et al., 1988). The pathway that processes ITD information is particularly well elucidated and the representation of ITD at each level of the pathway is orderly (Fig. 1). We therefore designed a visual manipulation that would induce a large change in the representation of ITD in the optic tectum with the intent of tracing the change to its point of origin.

We compared the representations of ITD in normal and prismreared owls at the level of the optic tectum and at the two prior stages of the ITD pathway: the central nucleus of the inferior colliculus (ICc) and the external nucleus of the inferior colliculus (ICx). We found that the representation of ITD in the ICc was unaffected by prism rearing, but that the representation in the ICx was shifted by an amount similar to that measured in the optic tectum. The results indicate that plasticity at the level of the ICx can account almost entirely for the visually guided changes in auditory spatial tuning measured in the optic tectum.

\section{Materials and Methods}

Visual experience. Six barn owls (Tyto alba) were raised with prismatic spectacles that displaced the visual field by $23^{\circ}$ either to the right or to the left with respect to the head. The experimental manipulation has been described in detail previously (Knudsen and Knudsen, 1989). Lightweight spectacle frames containing prismatic Fresnel lenses (40 diopters; Vision Care/3M) were sutured to the scalp of baby owls at 14-18 d of age, just as the eyes were beginning to open and before the ocular media had cleared. The prisms were cleaned daily and adjusted to maintain alignment with the owl's visual axes. At about $60 \mathrm{~d}$ of age, when the skull had reached adult size and hardened, permanent spectacles were mounted to a plate that was cemented to the skull.

The effect of the prisms on the visual field of each owl was measured by viewing the retinas ophthalmoscopically from a calibrated perimetry system. Zones of the visual field were defined as "deprived" if they were blocked by the spectacle frames or by the optics of the prisms, "displaced" if they were shifted by the prismatic lenses and were more than $10^{\circ}$ from the nearest deprived zone, or "marginal" if they were within $10^{\circ}$ of the deprived zone. Because the barn owl's eyes are essentially immobile with respect to the head, these zones corresponded to constant regions of the visual field. The displaced region of the visual field was centered within a few degrees of the owls' visual axes. The size of the displaced region ranged from $44^{\circ}$ to $60^{\circ}$ in azimuth and from $45^{\circ}$ to $56^{\circ}$ in elevation.

Owls were raised in an environment rich in auditory and visual stimuli. Babies were kept with siblings in brooding boxes where they vocalized and interacted regularly with each otber. As their primary feathers grew in, they were transferred to flight cages containing several other owls. Owls wearing spectacles showed a qualitatively similar degree of activity to owls with normal vision.

Acoustic measurements. The ITD cues available to owls were measured as a function of sound source location using methods described in detail previously (Olsen et al., 1989). Broad-band sounds were broadcast from a movable free-field speaker and the signals produced in the ear canals were recorded using probe tubes $(30 \mathrm{~mm} \times 2 \mathrm{~mm}$ o.d., 1 $\mathrm{mm}$ i.d.) attached to $1 / 2$ inch Bruel and Kjaer microphones. ITDs as a function of frequency were calculated using a fast Fourier transform spectrum analyzer and corrected for differences between the probe tubes. Contour plots of the variation of ITD with stimulus location were produced based on measurements made for sounds from 178 locations, spaced every $10^{\circ}$ in azimuth and elevation, across the frontal hemifield.

Electrophysiology. Electrophysiological measurements were made from owls after at least $150 \mathrm{~d}$ of prism rearing. During surgery, owls were anesthetized with halothane and nitrous oxide and all incisions werc infiltrated with lidocaine hydrochloride. Prior to the first recording session, a stainless steel plate, for securing the head during experiments, was cemented to the base of the skull and a craniotomy was opened over the approximate location of the optic tectum and inferior colliculus. The owl was wrapped in a soft leather jacket and suspended in a prone position in a sound isolation chamber. The head was bolted to a stereotaxic frame and aligned using retinal landmarks so that the visual axes lay within a horizontal plane. Anesthesia was maintained throughout the experiment with halothane and nitrous oxide or, in some cases, periodic injections of ketamine hydrochloride $(5-10 \mathrm{mg} / \mathrm{kg}$ ). At the end of a recording session the craniotomy was sealed with dental acrylic, fluids were administered intraperitoneally, and the owl was monitored in an observation cage until fully recovered.

Single and multiunit recordings were made from the optic tectum and inferior colliculus with insulated stainless steel or tungsten microelectrodes. A level discriminator was used to isolate units, and the timing of spikes (extracellular action potentials) was stored on a computer. Electrode penetrations were made in the vertical plane, perpendicular to the plane containing the owl's visual axes. The position of the electrode could be controlled in steps of $100 \mu \mathrm{m}$ in the horizontal plane and in steps of $1 \mu \mathrm{m}$ along the axis of the electrode.

Results are based primarily on extensive measurements from eight normal and four prism-reared owls. Measurements made in other studies of ITD tuning in the optic tectum are included from 10 additional normal owls. Two additional owls were raised with prismatic glasses but are not discussed: for one owl, preliminary measurement of auditory tuning in the tectum revealed an effect of laterally displaced vision on only a small portion of the tectum. For the second owl, there was a dramatic effect of visual displacement on auditory spatial tuning, but eye alignment was highly abnormal. Since the primary purpose of these experiments was to determine the site of change in the ascending auditory pathway that was responsible for the changes observed in the optic tectum, rather than to characterize the variability of the effects of prism rearing, only the four owls for which eye position was normal and auditory tuning in the tectum was shifted were studied in detail. Data from free-field studies of two of the prism-reared owls ( $\mathrm{Ll}$ and R1) have appeared previously (Knudsen and Brainard, 1991).

Auditory measurements. Auditory stimuli were generated digitally and delivered dichotically via matched earphones (Knowles, ED-1941 coupled to damping assembly BF-1743) placed in the external ear canals at a distance of about $5 \mathrm{~mm}$ from the eardrum. "Broad-band" stimuli were high-pass filtered at $4 \mathrm{kHz}$ to minimize sound propagation through the owl's interaural canal (Moiseff and Konishi, 1981b; Olsen et al., 1989).

Unit tuning was measured by presenting a series of noise bursts in which the ITD or ILD was varied in a randomized order, or a series of tones in which the frequency was varied in a randomized order. For each tuning measurement, 10-20 repetitions of a series were presented. All stimuli were $50 \mathrm{msec}$ in duration, with rise/fall times of $0 \mathrm{msec}$ for noise bursts and $5 \mathrm{msec}$ for tones, delivered at a rate of $1 / \mathrm{sec}$ and at an average binaural level of $20-30 \mathrm{~dB}$ above threshold for most units. Unit responses to stimuli were quantified as the number of spikes occurring in the $100 \mathrm{msec}$ following stimulus onset minus the number of spikes occurring in the $100 \mathrm{msec}$ prior to the stimulus (spontaneous rate). Responses to stimuli were interpolated between measurements made at intervals of $10-20 \mu \mathrm{sec}$ (ITD), $2-6 \mathrm{~dB}$ (ILD), or $0.25-1.0 \mathrm{kHz}$ (frequency). The width of tuning for a parameter was defined as the range of values for which the unit's interpolated response exceeded $50 \%$ of the maximum response. Best ITD and best ILD were defined as the midpoint of this range. ITD tuning was measured with ILD held constant at its best value, and ILD tuning was measured with ITD held constant at its best value. Best frequency was measured with both ITD and ILD held constant at their best values.

Units in the ICc are narrowly tuned to frequency, and sensitive to the relative phase of the signals at the two ears. Consequently, they respond similarly to values of ITD differing by integer periods of the unit's best frequency (Wagner et al., 1987; Fujita and Konishi, 1991). The best ITD for individual units in the ICc was therefore ambiguous. However, within a dorsoventral penetration, which passes from lowto high-frequency representations in the ICc (Knudsen and Konishi, 1978), there was typically a single value of ITD that was close to optimal for all units - the "array-specific ITD" (Wagner et al., 1987). For units in the ICc, we therefore measured best ITD for the peak in the response that was closest to the array-specific ITD. For a few penetrations there were abrupt changes in ITD tuning with depth such that no array-specific ITD was apparcnt. For these penetrations, best ITD was not detcrmined.

Latencies. Latencies to the onset of responses were determined using broad-band stimuli with optimal ITD and ILD presented at 20-30 dB 


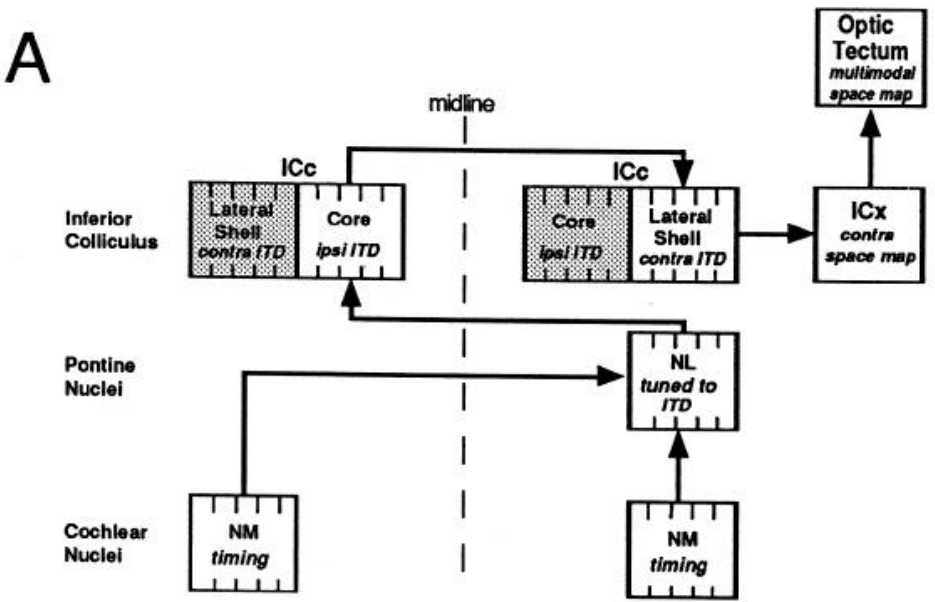

B
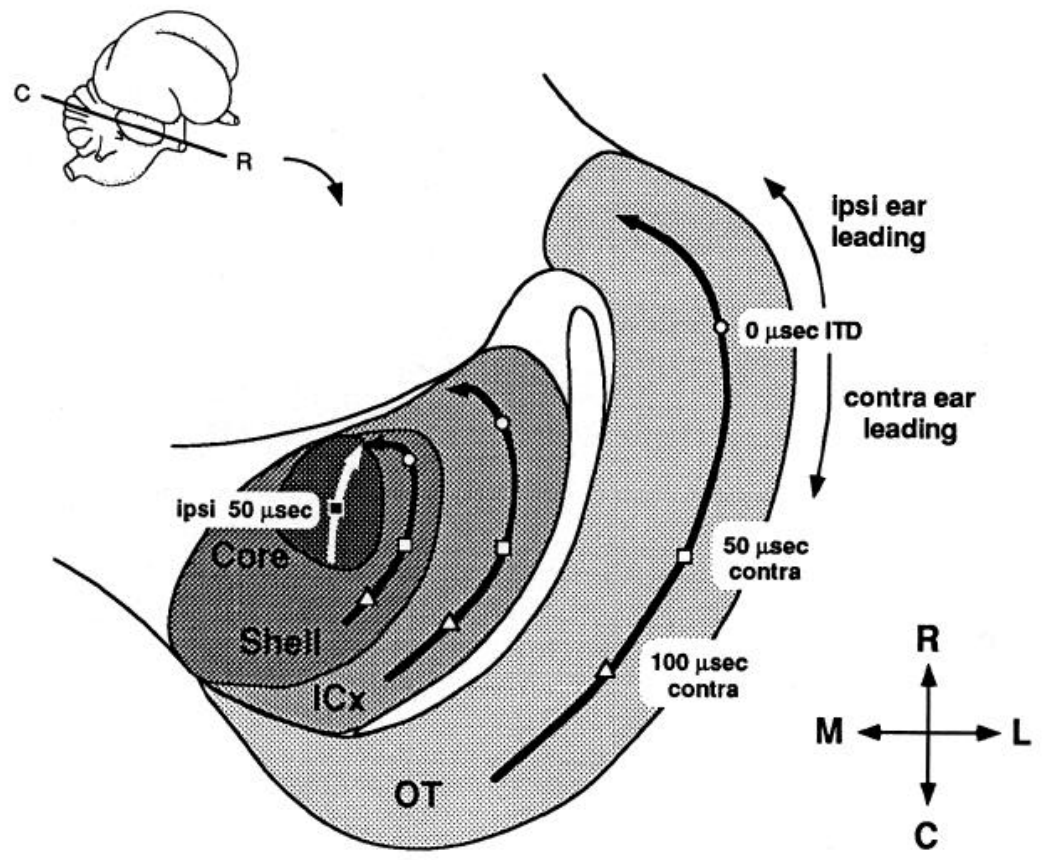

Figure 1. ITD pathway. A, Block diagram illustrating the main nuclei in the ascending ITD pathway to the optic tectum. All nuclei and connections are bilaterally symmetric, but are illustrated for only one side. Vertical tick marks indicate nuclei that are organized tonotopically. Each magnocellular cochlear nucleus $(N M)$ projects bilaterally to nucleus laminaris $(N L$; functionally equivalent to the mammalian medial superior olive), where the relative phases of the signals at the two ears are compared within narrow frequency channels (Sullivan and Konishi, 1986; Carr and Konishi, 1988, 1990). Nucleus laminaris projects contralaterally to the core of the central nucleus of the ICc (Takahashi and Konishi, 1988), which in turn projects back across the midline to the ICc lateral shell (Takahashi et al., 1989). The ICc lateral shell projects to the ICx (Knudsen, 1983; Wagner et al., 1987), and the ICx projects topographically to the optic tectum (Knudsen and Knudsen, 1983). B, Schematic diagram of a horizontal section through the right optic lobe, showing the representation of ITD in the optic tectum $(O T), \mathrm{ICx}$, ICc lateral shell, and ICc core. The plane of section is parallel to the dorsal surface of the optic tectum (inset). Best ITD varies along the rostrocaudal axis of each nucleus, with values corresponding to frontal locations represented rostrally. In the optic tectum, ICx, and ICc lateral shell, best ITDs become progressively more contra-ear-leading at locations farther caudal (Moiseff and Konishi, 1981a; Wagner et al., 1987; Olsen et al., 1989). In the ICc core, best ITDs become progressively more ipsi-earleading at locations farther caudal (Wagner et al., 1987). above threshold. The timing of spikes was recorded for each stimulus presentation, and peristimulus time histograms with $1 \mathrm{msec}$ bins were constructed from responses to at least 20 presentations. The first occurrence of two successive $1 \mathrm{msec}$ intervals in which the number of spikes exceeded the spontaneous rate of firing by 2 standard deviations of the spontaneous rate was found, and the latency was defined as the onset of the first of these intervals.

Visual measurements. For units in the optic tectum, visual receptive fields (VRFs) were mapped by projecting dark or light bars onto a calibrated translucent hemisphere $(57 \mathrm{~cm}$ in radius) placed in front of the owl. Locations are reported in a double-pole coordinate system (Knudsen, 1982) in which azimuth indicates degrees right (R) or left (L) of the midsagittal plane and elevation indicates degrees above $(+)$ or below $(-)$ the visual (horizontal) plane, all angles measured from the center of the head. At some recording sites, VRFs were not measured. For those sites, VRF azimuths were estimated based on interpolation between the VRF azimuths of units encountered more dorsally and more ventrally in the same penetration. VRF azimuth varies slowly and systematically with depth in a dorsoventral penetration and this pro- cedure was found to be accurate to within about $1^{\circ}$ when tested on units with measured VRFs.

Histology. Marking lesions were made by passing 3-5 $\mu \mathrm{A}$ of cathodal current for 10-20 sec. After 2-10 d survival times, owls were given an overdose of sodium pentobarbital $(300 \mathrm{mg} / \mathrm{kg})$ and perfused with either $4 \%$ paraformaldehyde or $10 \%$ formalin. Frozen sections were cut at 40 $\mu \mathrm{m}$ in a horizontal plane parallel to the dorsal surface of the optic tectum, and every third section was Nissl stained to identify lesions. Sections containing the IC were also stained with an antibody that marks the ICc core by labeling a calcium-binding protein-like antigen in the terminal field of the projection from nucleus laminaris (Takahashi et al., 1987). The antibody (7E4 F2) was supplied by C. E. Carr.

Targeting of nuclei. A variety of stereotaxic and physiological criteria was used during recording sessions to target and identify the optic tectum and the central and external subdivisions of the inferior colliculus. In four control owls and two experimental owls, lesions were placed in these nuclei in order to provide an anatomical confirmation of physiologically determined recording sites.

In each recording session, the first penetrations were made in the optic 


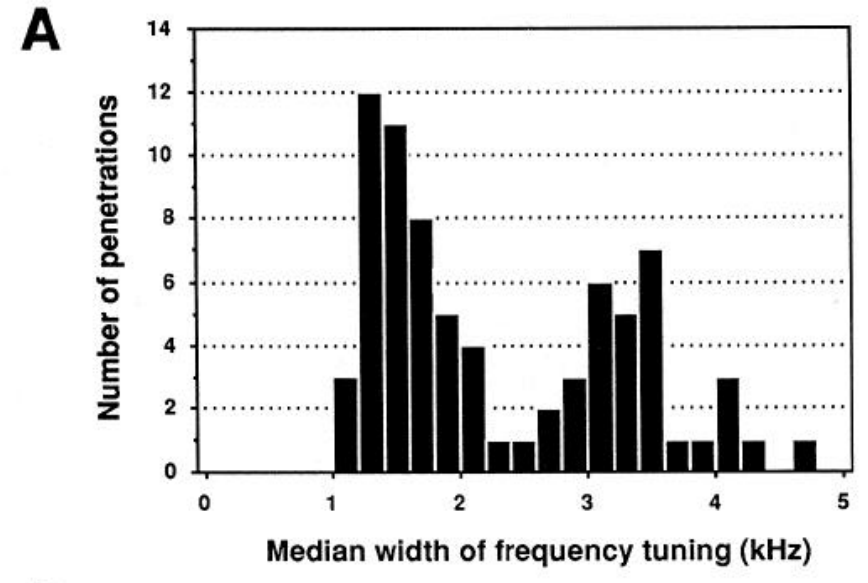

B

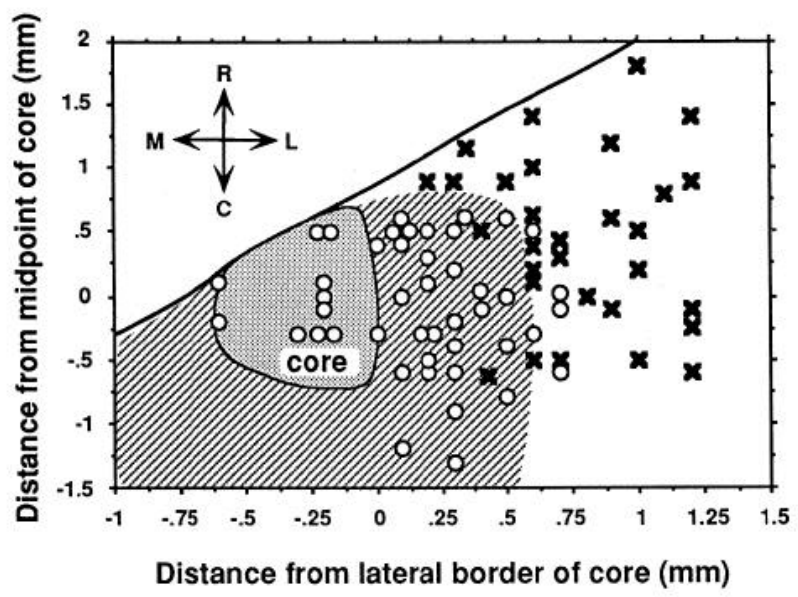

Figure 2. Width of frequency tuning as a criterion for distinguishing between the ICc and the ICx. A, Histogram of the median width of frequency tuning for 75 penetrations through seven inferior colliculi in four normal owls. The distribution was bimodal, with peaks centered at approximately $1.5 \mathrm{kHz}$ and $3.3 \mathrm{kHz}$. $B$, Locations relative to the ICc core at which broad and narrow frequency tuning were recorded. Locations, in the horizontal plane, of the penetrations from $A$ are plotted relative to the rostrocaudal midpoint of the lateral edge of the ICc core. Locations were reconstructed based on lesions or stereotaxic coordinates of penetrations relative to sites at which lesions were made. The approximate region of core staining for a typical IC is indicated by stippling. Penetrations in which sharp frequency tuning was observed (median width $<2.4 \mathrm{kHz}$; circles) were located primarily within $500 \mu \mathrm{m}$ of the core, while those in which broad frequency tuning was observed (median width $>2.4 \mathrm{kHz} ; X s$ ) tended to be located farther lateral. The regions of sharp and broad frequency tuning were assigned to the ICc and $\mathrm{ICx}$, respectively.

tectum. Characteristic "bursty" activity and the presence of spatially restricted receptive fields for visual and auditory stimuli confirmed that the electrode was in the optic tectum. By comparing unit visual receptive fields to the published map of space in the optic tectum (Knudsen, 1982; Olsen et al., 1989), subsequent penetrations could then be directed accurately either to other locations in the optic tectum or to the inferior colliculus.

The ICC was distinguished from the ICx based on unit tuning to frequency and to ILD. In the ICx, units are tuned for auditory stimulus location and organized into a map of space; frequency tuning is broad, and best ILDs progress from right-ear-greater to left-ear-greater with depth (Knudsen and Konishi, 1978; Knudsen, 1984; Mogdans and Knudsen, 1990). In the ICc, units are sharply tuned for frequency, best frequencies progress systematically from low to high with depth, and there is not typically a systematic progression of best ILDs (Knudsen and Konishi, 1978; Knudsen, 1984; Wagner et al., 1987). The median width of frequency tuning measured for a dorsoventral penetration through the IC was a reliable criterion for physiologically distinguishing
Table 1. Sharpness of tuning for ITD and ILD

\begin{tabular}{lllllll} 
& & \multicolumn{2}{c}{ L23 prisms $^{b}$} & & \multicolumn{2}{c}{ R23 prisms $^{b}$} \\
& Normal $^{a}$ & L1 & L2 & & R1 & R2 \\
\hline ITD 50\% width $(\mu \mathrm{sec})$ & & & & & \\
Mean & 42.1 & 45.0 & 37.4 & & $52.0^{*}$ & $44.9^{c}$ \\
SD & 14.9 & 11.9 & 12.8 & 25.0 & 12.8 \\
$n$ & 126 & 22 & 37 & 33 & 42
\end{tabular}

ILD $50 \%$ width $(\mathrm{dB})$

$\begin{array}{lccccc}\text { Mean } & 12.2^{d} & 11.2 & 9.7^{*} & 13.6 & 9.8^{*} \\ \text { SD } & 4.3 & 2.3 & 3.7 & 3.6 & 3.9 \\ n & 111 & 16 & 34 & 17 & 37\end{array}$

a Data from 18 owls at recording sites with VRFs centered between $\mathrm{L} 20^{\circ}$ and R20 azimuth and between $-30^{\circ}$ and $+20^{\circ}$ elevation.

${ }^{b}$ Recording sites with VRFs that were prismatically displaced.

c At four sites, ITD tuning curves were abnormally shaped, with more than one local maximum in plots of response as a function of ITD; these were excluded from calculation of the mean.

${ }^{d}$ At five sites, unit responses were greater than $50 \%$ of the maximum for the most extreme values or right-ear-greater ILD which were tested $(+30 \mathrm{~dB})$; these were excluded from calculation of the mean.

* Significantly different from normal (ANOVA, $p<0.01$ ).

between the ICc and ICx (Fig. 2). Median widths of frequency tuning formed a bimodal distribution (Fig. $2 A$ ), and penetrations associated with narrow frequency tuning were consistently found medial to penetrations with broader frequency tuning. Anatomical reconstructions revealed that nearly all penetrations that had median widths of frequency tuning less than $2.4 \mathrm{kHz}$ were found within $600 \mu \mathrm{m}$ of the ICc core (Fig. 2B). This region is consistent with prior physiological and anatomical characterizations of the extent of the ICc (Wagner et al., 1987; Takahashi and Konishi, 1988; Takahashi et al., 1989).

\section{Results}

The representations of ITD in the optic tectum, ICx, and ICc were assessed in normal owls and in four experimental owls: two raised with prismatic glasses that shifted the visual field $23^{\circ}$ to the left (owls L1 and L2), and two raised with glasses that shifted the visual field $23^{\circ}$ to the right (owls R1 and R2). Measurements were made after at least $150 \mathrm{~d}$ of prism rearing, and therefore indicate the final representation of ITD established in each of the nuclei. The results for each nucleus are presented separately below.

\section{The optic tectum}

Representation of interaural time difference. The representation of ITD in the optic tectum was assessed by comparing the best ITDs of units with the locations of their VRFs. In normal owls, as reported previously (Olsen et al., 1989), units were sharply tuned for ITD (Table 1), and the values of ITD to which units responded best corresponded to the values of ITD produced by sounds at the locations of their VRFs. For example, Figure $3 A$ shows a contour plot of the ITD cues measured in an owl's ear canals as a function of the location of a sound source in the frontal hemifield. Each line indicates locations from which a constant value of ITD was produced: ITD changed little with elevation, but varied systematically with the azimuth of a sound source. Superimposed on the plot is the location of the VRF recorded from a unit in the optic tectum of a normal owl. The VRF was located directly in front of the owl, at a location from which sound sources produced an ITD of about $0 \mu \mathrm{sec}$. The auditory tuning of the unit for ITD is shown in Figure $3 B$. The unit responded most strongly to the value of ITD produced by 
A

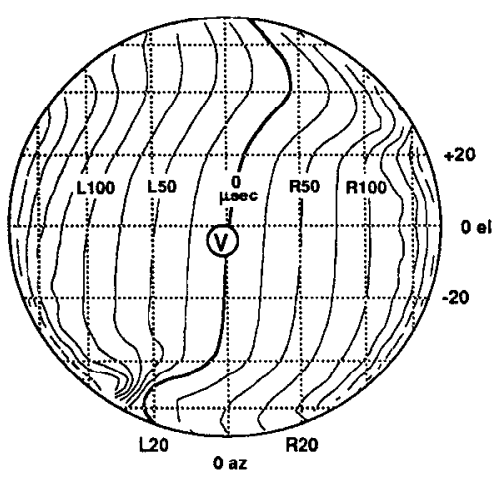

C

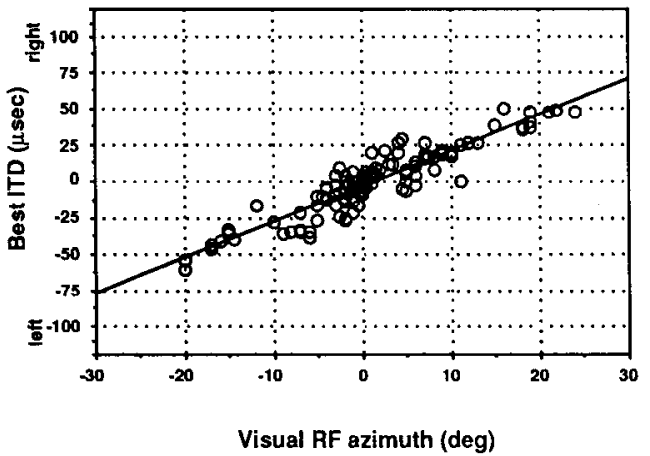

B

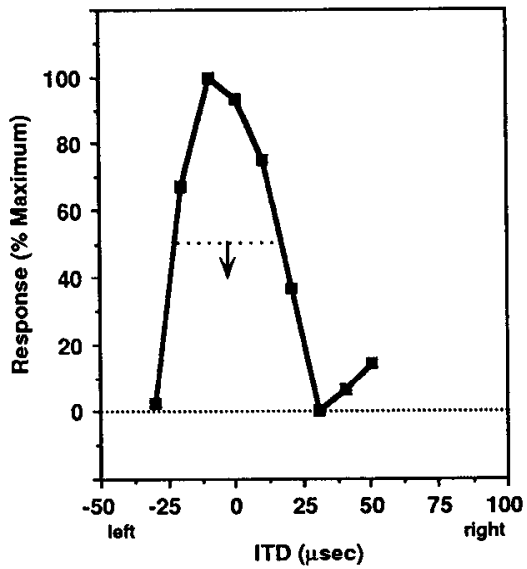

D

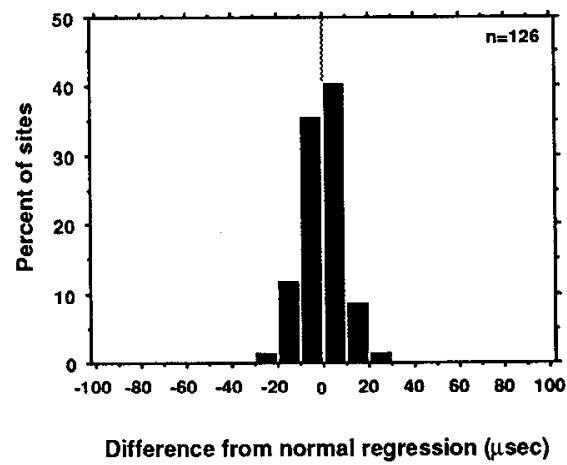

Figure 3. Normal correspondence between VRF location, acoustic cues, and unit tuning for ITD. $A$, The VRF location of a unit recorded from the optic tectum of a normal owl (encircled $V$ ) is shown on a representation of the frontal hemifield. Superimposed is a contour plot of the values of ITD (at $6.3 \mathrm{kHz}$ ) produced in the ear canals of a barn owl as a function of the location of a freefield sound source; the ITD contours are based on an average of measurements made from five normal adult barn owls. $B$, Normalized response as a function of stimulus ITD is plotted for the same unit for which the VRF is indicated in $A$. The unit had a best ITD close to $0 \mu \mathrm{sec}$ (downward arrow), matching the ITD produced by a sound source at the location of its VRF. $C$, Best ITD is plotted as a function of VRF azimuth for 126 sites from 18 normal owls. Analysis was restricted to sites where VRF centers were located between $\mathrm{L} 25$ and R25 degrees in azimuth and $+20^{\circ}$ and $-30^{\circ}$ in elevation. The solid line is a linear least-squares fit to the data $\left(y=2.48 x-2.7 ; r^{2}=0.841\right)$. $D$, Histogram of the residuals for the data plotted in $C$. a sound source at the location of the VRF $(0 \mu \mathrm{sec})$. The relationship between best ITD and VRF azimuth for 126 units from normal owls is summarized in Figure $3 C$. Unit best ITDs varied systematically with VRF azimuth, from left-ear-leading ITDs to right-ear-leading ITDs as VRFs progressed from left to right. A linear least-squares fit to the data had a slope of $2.48 \mu \mathrm{sec} /$ degree and an intercept close to $0 \mu \mathrm{sec}$ at $0^{\circ}$ azimuth. The differences between measured values of best ITD and values predicted from the fit to the normal data are plotted in Figure $3 D$.
For the majority of sites, the differences were less than $10 \mu \mathrm{sec}$, indicating that VRF azimuth accurately predicts unit tuning for ITD in normal owls.

The prismatic glasses worn by owls optically shifted a large portion of the visual field by $23^{\circ}$ along the horizon, but had little effect on the localization cues reaching the ears (Knudsen and Knudsen, 1989). Consequently, the prisms altered the normal associations between auditory cues and locations in the visual field. For example, for a normal owl, an object that ap-
A Apparent location: $0^{\circ}$
Actual location: $0^{\circ}$ ITD: 0 usec

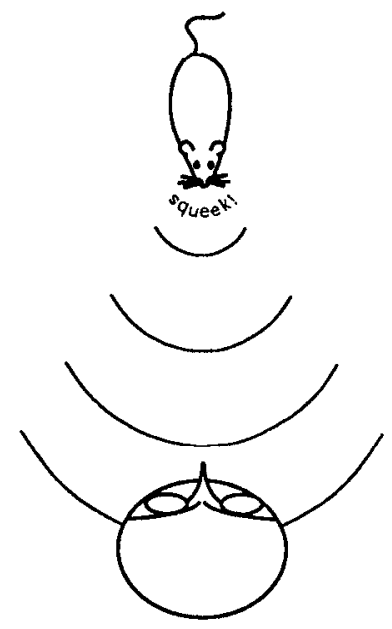

Normal
B Apparent location: $0^{\circ}$
Actual location: $23^{\circ}$ right

ITD: rightear-leading

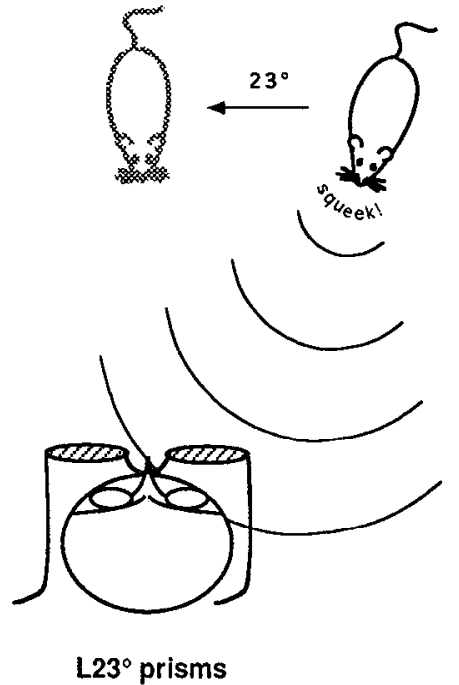

Figure 4. Effect of $\mathrm{L} 23^{\circ}$ prisms on the correspondence between auditory cues and apparent locations of sound sources. $A$, Normally an object that appears to be directly in front of the owl produces sounds that reach the two ears simultaneously. $B$, For an owl wearing L $23^{\circ}$ prisms, an object that appears to be directly in front of the owl is actually located $23^{\circ}$ to the owl's right, and produces sounds that reach the right ear before the left. 
A

Figure 5. Effect of prism rearing on unit tuning for ITD. $A$, VRF of a unit recorded in the optic tectum of an owl reared with $\mathrm{L} 23^{\circ}$ prisms is shown on a representation of the frontal hemifield. Also indicated is the variation of acoustic cues with location (replotted from Fig. $3 A$ ). The VRF, measured without prisms (open circle), was centered at $\mathrm{L} 1^{\circ}$ azimuth and $-5^{\circ}$ elevation. The shaded circle shows the location to which the VRF was optically displaced while prisms were worn. $B$, Normalized response is plotted as a function of stimulus ITD for the same unit for which the VRF is indicated in $A$. The unit had a best ITD (downward arrow) of $52 \mu \mathrm{sec}$ right-ear-leading. The ITD tuning of a unit that had a VRF at the same location, but that was recorded from a normal owl, is shown for comparison (shaded line, replotted from Fig. 3B). $C$, Best ITD is plotted as a function of VRF azimuth for 59 sitcs from two owls reared with $\mathrm{L} 23^{\circ}$ prisms (squares, owl L1; circles, owl L2). Analysis was restricted to sites where VRFs were optically displaced as determined by perimetry measurements (see Materials and Methods). The best fit to the normal data (shaded line) is plotted for comparison. $D$, Histogram of differences between measured values of best ITD for owls L1 and L2 (solid bars) and values predicted from the normal relationship between best ITD and VRF azimuth (open bars). $E$, Best ITD is plotted as a function of VRF azimuth for 74 sites from two owls reared with $\mathrm{R} 23^{\circ}$ prisms (squares, R1; circles, R2). $F$, Histogram of differences between measured values of best ITD for owls $\mathrm{R} 1$ and R2 (solid bars) and values predicted from the normal relationship between best ITD and VRF azimuth (open bars).

C

E
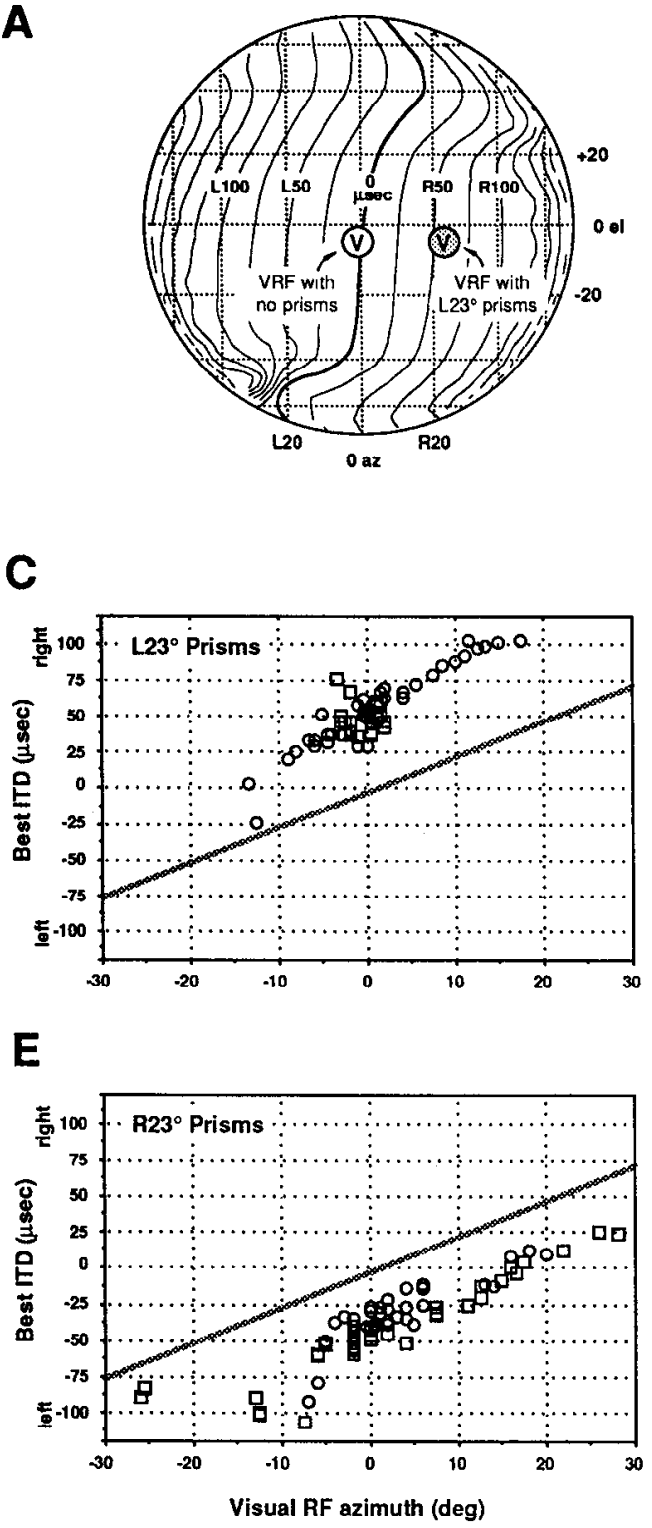

B

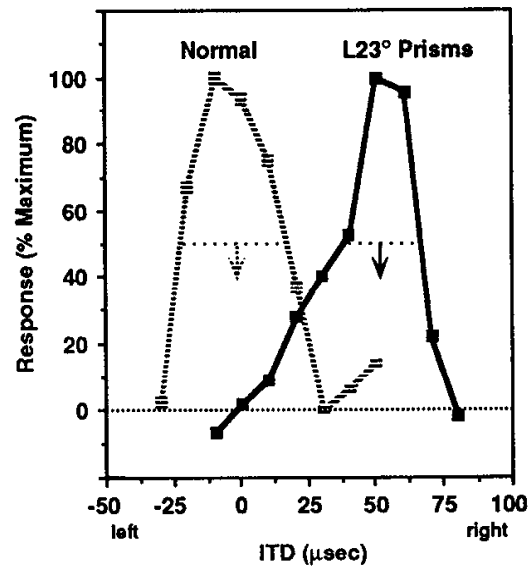

D

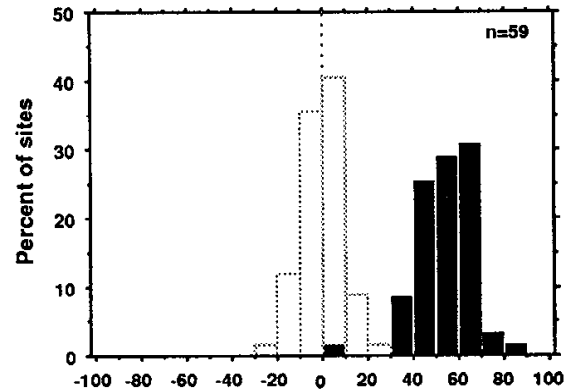

$\mathbf{F}$

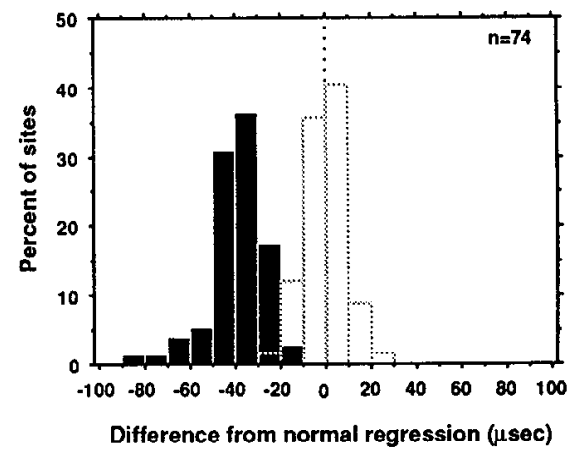

pears to be directly in front of the owl, at $0^{\circ}$ azimuth, produces an ITD of about $0 \mu \mathrm{sec}$ (Fig. $4 A$ ). However, for an owl wearing prisms that shift the visual field $23^{\circ}$ to the left, an object that appears to be at $0^{\circ}$ azimuth is actually located $23^{\circ}$ to the right, and produces sounds that arrive at the right ear before the left (Fig. 4B). Prismatic glasses alter the correspondence of ITD values with unit VRF locations in an analogous fashion: Figure $5 A$ shows the optical effect of prisms on the VRF of a unit recorded from an owl that was raised wearing $L 23^{\circ}$ prisms. The unit had a VRF, measured without prisms, directly in front of the owl, at a location where sounds produced an ITD of $0 \mu \mathrm{sec}$ (open circle). However, when $\mathrm{L} 23^{\circ}$ prisms were placed on the owl, the VRF of the same unit was optically shifted $23^{\circ}$ to the right, to a location where sounds produced an ITD of about 60 $\mu$ sec right-ear-leading (shaded circle). Conversely, in owls raised wearing $\mathrm{R} 23^{\circ}$ prisms, VRFs were chronically shifted by $23^{\circ}$ to the left, and consequently corresponded with more left-ear-leading ITDs than normal.

In prism-reared owls, units were tuned to values of ITD corresponding with sound sources at the location of their optically displaced VRFs and not to the values appropriate for their VRFs measured without prisms. For example, Figure $5 B$ shows the ITD tuning of the unit for which VRFs are indicated in Figure $5 A$. This unit had a VRF, measured without prisms, located directly in front of the owl, and in a normal owl it would have responded most strongly to an ITD of about $0 \mu \mathrm{sec}$ (shaded tuning curve). However, this unit did not respond at all to an ITD of $0 \mu \mathrm{sec}$, and instead had a best ITD of $52 \mu \mathrm{sec}$ right-earleading (solid tuning curve). Because such shifts bring a unit's best ITD tuning closer to the value produced by sounds at the location of the prismatically displaced VRF, they will be referred to as "adaptive" shifts in ITD tuning.

The effect of prism rearing on the correspondence between best ITD and VRF azimuth for all units that were subjected to displaced VRFs $(n=138)$ is summarized in Figure $5 C-F$. Units were generally sharply tuned for ITD (Table 1). However, at four sites ITD tuning curves were abnormally shaped, and these sites were excluded from further analysis. In each of the prismreared owls, best ITD varied systematically with azimuth, as in normal owls, but over a large range of VRF azimuths, the re- 


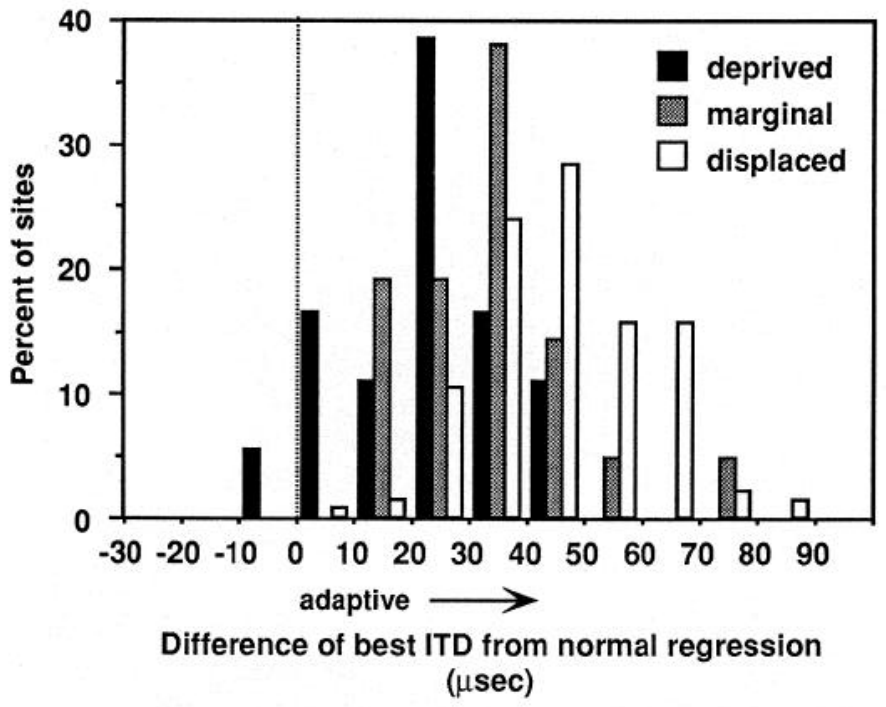

Figure 6. Summary of shifts in best ITD for recording sites where either the deprived, marginal, or displaced zones were represented. Shifts in best ITD were calculated as the difference between measured values of best ITD and values predicted from VRF location based on a linear fit to data from normal owls (Fig. $3 C$ ).

lationship was shifted in the direction of VRF displacement: toward right-ear-leading ITDs for owls reared with $\mathrm{L} 23^{\circ}$ prisms (Fig. 5C) and toward left-ear-leading ITDs for owls reared with $\mathrm{R}^{2} 3^{\circ}$ prisms (Fig. $5 E$ ). The amount of shift for each site was quantified (as it was for data from normal owls, Fig. $3 D$ ) as the difference between the measured value of best ITD and the value predicted from a linear regression of the normal data (Fig. $5 D, F$ ). The median shifts in best ITD for owls L1, L2, R1, and R2 were $51,55,43$, and $37 \mu \mathrm{sec}$, respectively.

Regions of the optic tectum that represented parts of the visual field that were blocked by the spectacle frames ("deprived") or were at the border of deprived and displaced zones ("marginal") also had shifted representations of ITD (Fig. 6). At six sites in the marginal zone, ITD tuning curves were abnormally shaped, and these sites were excluded from further analysis. The median adaptive shifts for sites in the deprived $(n=18)$ and marginal $(n=21)$ zones (pooled across all owls) were 24 and $34 \mu \mathrm{sec}$, respectively. These shifts were smaller than the median shift of $44 \mu \sec (n=134)$ measured for the displaced zone, but were significantly different from normal (ANOVA, $p<0.001$ ).

Several lines of evidence argue that the abnormal relationship between auditory and visual spatial representations is due to a shift in the auditory map rather than the visual map: The azimuths of VRFs varied little within dorsoventral penetrations, while ITD tuning in many penetrations varied abruptly at the border between visually deprived and displaced zones. Further, in some tecta, values of best ITD up to $60 \mu \mathrm{sec}$ ipsi-ear-leading were recorded. Such large ipsi-ear-leading values are normally not found in the optic tectum (Olsen et al., 1989). They are consistent with the prior observation in prism-reared owls of units with auditory receptive fields centered at azimuths outside the normal range (Knudsen and Brainard, 1991).

To ascertain the magnitude of the shift in the ITD map across the optic tectum, electrolytic lesions were placed at physiologically characterized sites in six tecta from four normal owls and in three tecta from two prism-reared owls (Fig. 7). In normal owls, VRF azimuth and best ITD varied systematically in the
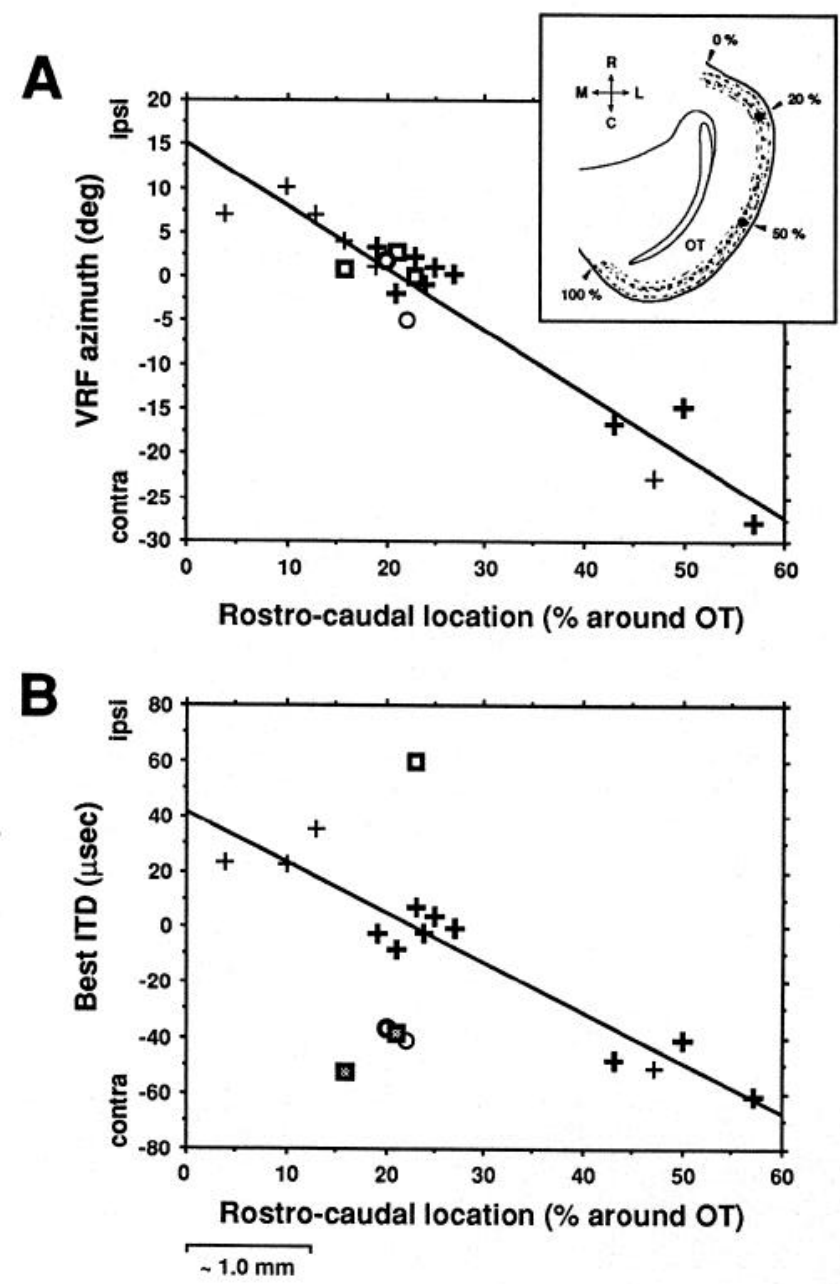

Figure 7. Variation of VRF azimuth and best ITD with anatomical location in the optic tectum for normal and prism-reared owls. $A$, VRF azimuth is plotted as a function of anatomical location in the optic tectum for recording sites from an owl raised with $\mathrm{L}^{2} 3^{\circ}$ prisms (squares, L1), an owl raised with $\mathrm{R} 23^{\circ}$ prisms (circles, R1) and four normal owls (crosses). Recording sites were either determined by recovery of lesions (bold symbols) or were estimated based on their microdrive coordinates relative to recovered lesions. Data are pooled from the left and right tecta of owls, and VRF azimuths are expressed as degrees ipsilateral or contralateral to the side of recording. Locations are expressed as a percentage of the distance around the optic tectum $(O T)$ from the rostral to the caudalmost extent of the cell dense tectal layers visible in Nisslstained sections (inset). Measurements were made in horizontal sections cut parallel to the long axis of the optic tectum (Fig. $1 B$, inset). The solid line is a linear least-squares fit to the normal data. The data from the prism-reared owls fall close to the normal regression, indicating that the visual map in the rostral optic tectum was not shifted by prism rearing. $B$, Best ITD is plotted as a function of location in the optic tectum. Data are from the same recording sites as in $A$. The solid line indicates the linear least-squares fit to the data from normal owls (crosses). The values of best ITD recorded from prism-reared owls (circles and squares) were shifted relative to the regression of normal data by amounts ranging from 42 to $60 \mu \mathrm{sec}$.

horizontal plane, from values corresponding to frontal space at the rostral end of the optic tectum, to values corresponding to contralateral space at the caudal end of the optic tectum (crosses in Fig. 7). In the prism-reared owls, VRF locations (measured without prisms) were normal for recording sites at the rostral end of the optic tectum (circles and squares in Fig. $7 A$ ), confirming that prism rearing did not shift the visual map of space. 
However, at the same locations, the values of best ITD recorded from prism-reared owls were abnormal (circles and squares in Fig. $7 B$ ). In the right optic tectum of owl R1 (circles), the best ITD represented at the rostral end of the optic tectum was 40 $\mu \mathrm{sec}$ left-ear-leading (contra-ear-leading). This value of ITD is normally represented at approximately $50 \%$ of the distance around the optic tectum. In the right optic tectum of owl L1 (open square), the best ITD represented at a similar location was about $60 \mu \mathrm{sec}$ right-ear-leading (ipsi-ear-leading). This value of ITD cxcceds the most ipsilateral values of ITD normally represented at the rostral end of the optic tectum. In the same owl, the value of best ITD at $20 \%$ of the distance around the left optic tectum (shaded squares) was also shifted toward rightear-leading (contra-ear-leading), which on this side of the brain is normally represented farther caudal. These shifts correspond to displacements of the normal map of ITD by at least $25 \%$ of the distance around the optic tectum, or approximately $2 \mathrm{~mm}$.

Representation of interaural level difference. In the optic tectum of normal owls, best ILDs varied systematically with VRF elevation [best ILD (in $\mathrm{dB})=0.62 * \mathrm{VRF}$ elevation (in degrees) $+3.0, r^{2}=0.857$; least-squares linear fit to data from 111 sites from 18 normal owls]. Rearing owls with laterally displacing prisms had only a small effect on this relationship. For 104 sites where units were subjected to displaced VRFs, shifts in ILD tuning were quantified as differences between measured values of best ILD and values predicted from the normal relationship between best ILD and VRF elevation. The median shifts in best ILD for owls L1, L2, R1, and R2 were $0.6,0.1,5.0$, and 2.8 $\mathrm{dB}$, respectively, in the direction of the optical displacement of the VRF (i.e., toward left-ear-greater for $\mathrm{R} 23^{\circ}$ prisms and rightear-greater for $\mathrm{L}_{23^{\circ}}$ prisms). These values were significantly different from normal for owls R1 (ANOVA, $p<0.001$ ) and R2 $(p<0.001)$, but not for owls L1 $(p-0.48)$ and L2 $(p=$ $0.48)$.

\section{The central nucleus of the inferior colliculus}

The representation of ITD in the lateral shell and core of the ICc was assessed by making a series of closely spaced dorsoventral penetrations through the ICc and measuring the variation of best ITD with rostrocaudal position. The ICc is organized tonotopically with isofrequency laminae lying approximately in the horizontal plane (Knudsen and Konishi, 1978; Wagner et al., 1987). Penetrations traversed from low- to high-frequency representations, and best ITDs were determined for units in the $6 \mathrm{kHz}$ lamina. In four normal owls, lesions were placed bilaterally at selected recording sites in the $6 \mathrm{kHz}$ lamina so that the variation of ITD tuning relative to anatomical markers could be determined. The locations of histologically reconstructed recording sites were measured relative to the ICc core, which was stained immunohistochemically (Fig. $8 A$ ). Positions of penetrations in both the lateral shell and the core were quantified as percentage distance caudal relative to the rostrocaudal extent of core staining (Fig. $8 \mathrm{~B}$ ). Although similar results were obtained when other anatomical landmarks were used for reference, this measure yielded the most consistent representation of ITD in normal owls.

Best ITD varied systematically with location along the rostrocaudal axis of the ICc in normal owls (Fig. 9A,B). In the lateral shell, values of ITD corresponding to frontal spatial locations (about $0 \mu \mathrm{sec}$ ) were represented at the rostral end of the nucleus ( $0 \%$ caudal), and progressively more contra-ear-leading values of ITD were represented farther caudal. Within the core, best ITD values were progressively more ipsi-ear-leading at locations farther caudal. Together, the lateral shell and core contained a more or less continuous representation of ITD, such that when the representation was "unwrapped" as in Figure $9 B$, it could be fit well by a second-order polynomial: most best ITDs were within $20 \mu \mathrm{sec}$ of the values predicted by the fit (Fig. $9 B$, shaded region; $C$ ).

In the ICc of two prism-reared owls, the representation of ITD was determined in the same manner. The pattern of core immunohistochemical staining, used to quantify the locations of recording sites, did not itself differ between normal and prismreared owls (Table 2). Figure $9, D$ and $E$, compares the representations of ITD measured in the prism-reared owls with that found in normal owls. The solid lines and shaded region indicate the polynomial fit to the normal data. The dashed lines indicate the representation of ITD predicted if a shift at the level of the ICc were responsible for the shift measured in the optic tectum. For example (Fig. $9 E$ ), in an owl raised with $\mathrm{R} 23^{\circ}$ prisms, the value of ITD represented at the rostral end of the right ICc lateral shell (normally $0 \mu \mathrm{sec}$ ) would be $50 \mu \mathrm{sec}$ left-ear-leading (contra-ear-leading), corresponding to a rostralward displacement of the ITD map. Conversely, in an owl raised with L $23^{\circ}$ prisms, the value of ITD represented at the rostral end of the right ICc lateral shell would be $50 \mu \mathrm{sec}$ right-ear-leading (ipsiear-leading), corresponding to a caudalward displacement of the ITD map. Such shifts were not observed (Fig. 9D,E), indicating that the representation of ITD was essentially normal in both the core and the lateral shell.

The representation of ITD in prism-reared owls was compared quantitatively with the representation in normal owls by calculating differences between measured best ITDs and best ITDs predicted from the second-order polynomial regression of the normal data. Best ITD residuals of the normal data ranged between $\pm 30 \mu \mathrm{sec}$ and had a median value of $0 \mu \mathrm{sec}$ (Fig. 9C). For the penetrations from prism-reared owls, differences from the normal regression were assigned positive values if they were in the adaptive direction (appropriate to account for the shift observed in the optic tectum) and negative values if they were in the opposite direction. The values for all penetrations in the prism-reared owls fell within the normal range (shaded bars, Fig. $9 F$ ), and had a median adaptive shift of $4 \mu \mathrm{sec}$. This was not significantly different from normal, either when the data from the core and shell were pooled together ( $t$ test, $p=0.28$ ) or when they were considered individually.

In another four normal owls and two prism-reared owls, the representation of ITD in the ICc was mapped without making lesions (Fig. 10). In these owls, best ITD values were determined for a series of closely spaced ( $300-500 \mu \mathrm{m}$ separation) penetrations through the rostrolateral corner of the ICc. The rostral border of the ICc was determined based on physiological criteria (see Materials and Methods) and provided the reference for characterizing the representation of ITD in the ICc.

The results were consistent with those obtained in the lesion experiments described above. In normal owls, the rostralmost values of best ITD in the ICc were close to $0 \mu \mathrm{sec}$. For example, Figure $10 \mathrm{~A}$ shows the values of best ITD from a series of penetrations in the right ICc of a normal owl. The best ITD for the rostralmost penetration in the ICc was $11 \mu \mathrm{sec}$ ipsi-ear-leading. At locations farther caudal and lateral, best ITDs became progressively more contra-ear-leading. At locations farther caudal and medial, best ITDs became progressively more ipsi-ear-leading. In four ICc from four normal owls, best ITD values at the 


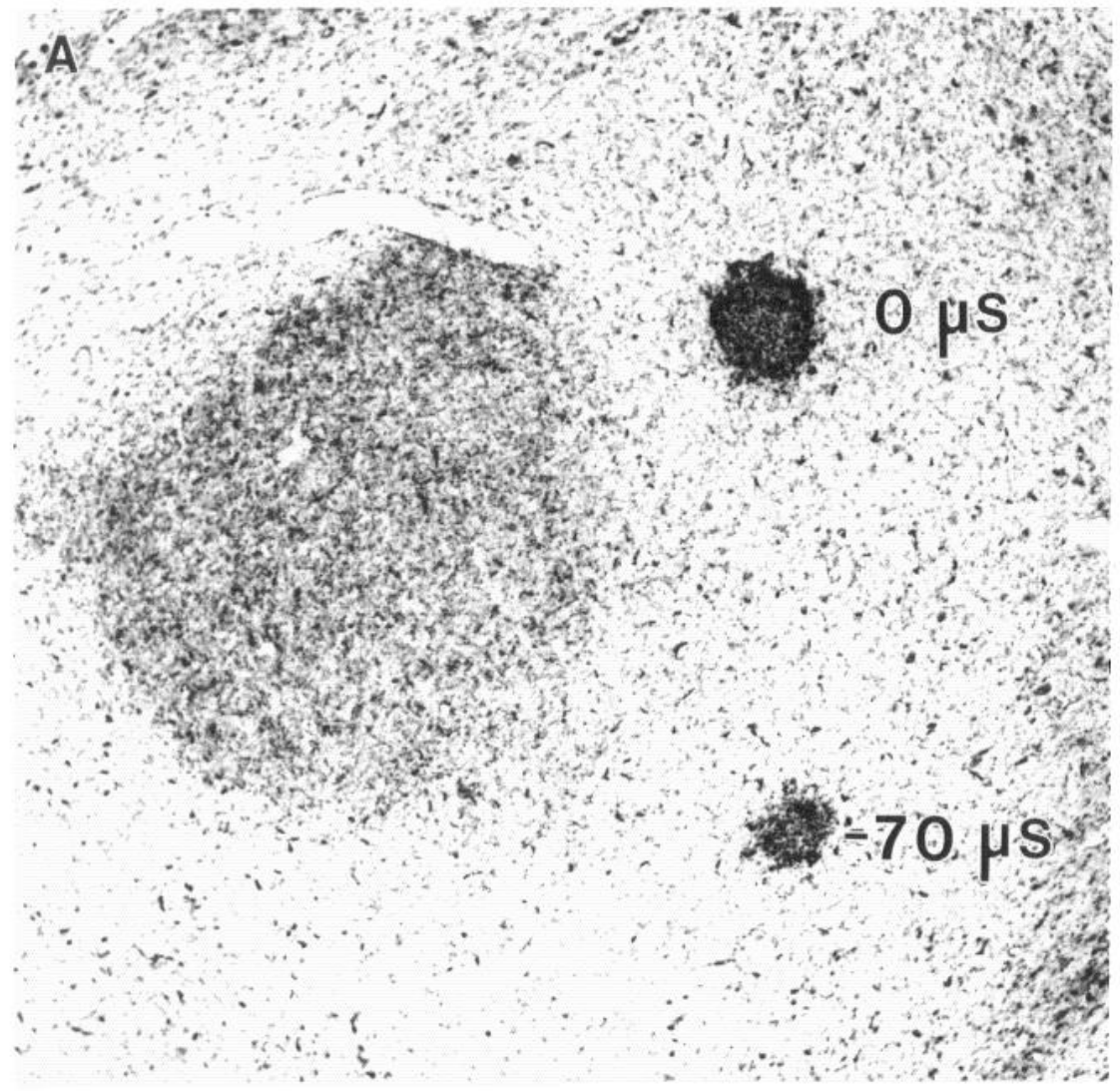

B<smiles>[M]C([R])([3H])C</smiles>

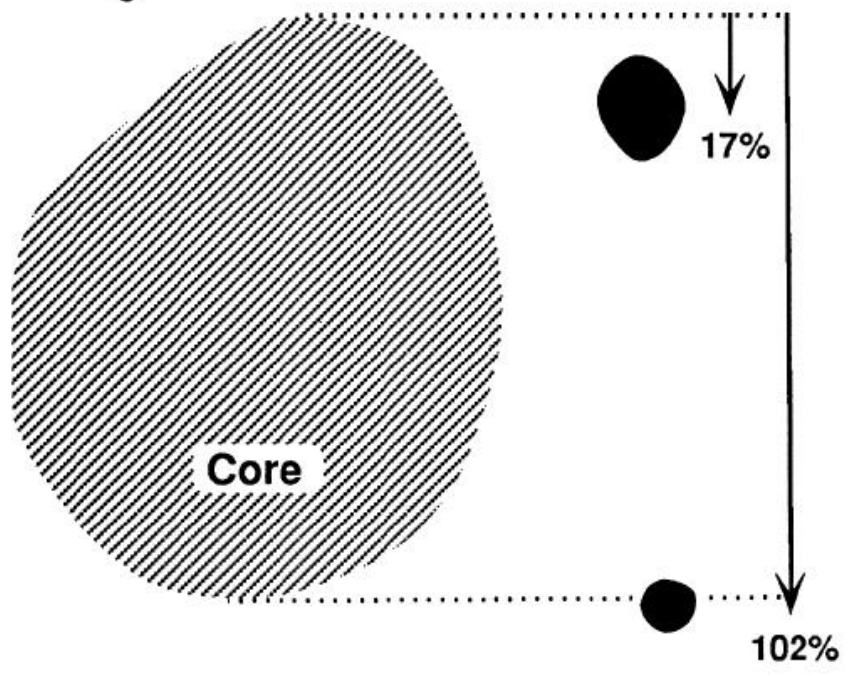

$500 \mu \mathrm{m}$
Figure 8. The core of the ICc as an anatomical marker for characterizing the locations of recording sites in the ICc. $A, \mathrm{~A}$ horizontal section through the right ICc showing the core immunohistochemically stained with an antibody to calbindin and counterstained with a Nissl stain. The region of staining corresponds to the terminal field of the projection from the contralateral nucleus laminaris(Takahashi et al., 1987). Numbers indicate the values of best ITD that were recorded at the lesion sites. $B$, Schematic of the section shown in $A$. 
A

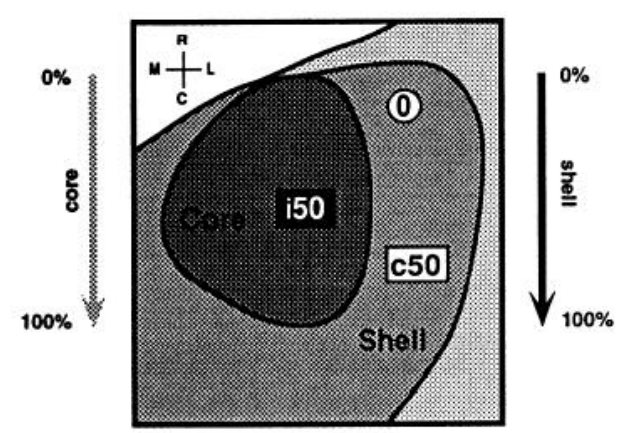

B

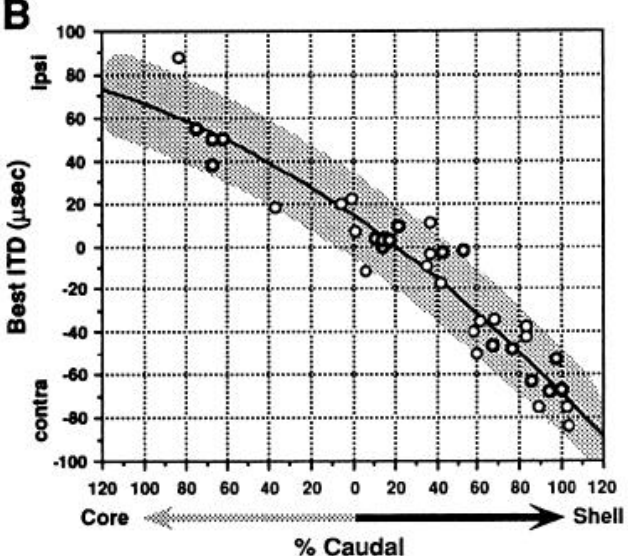

C

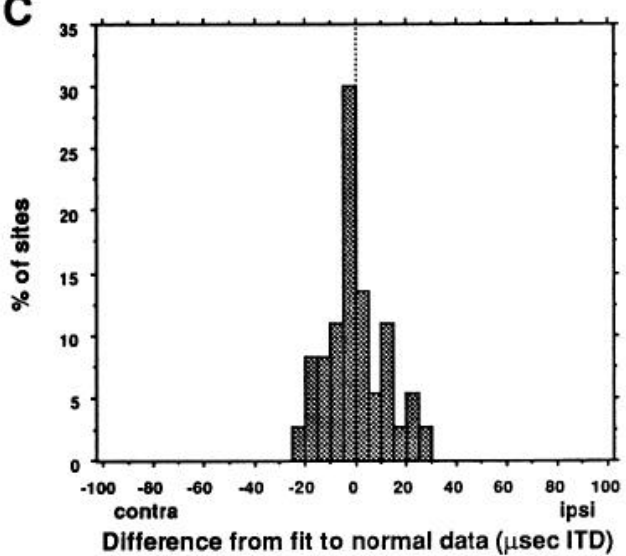

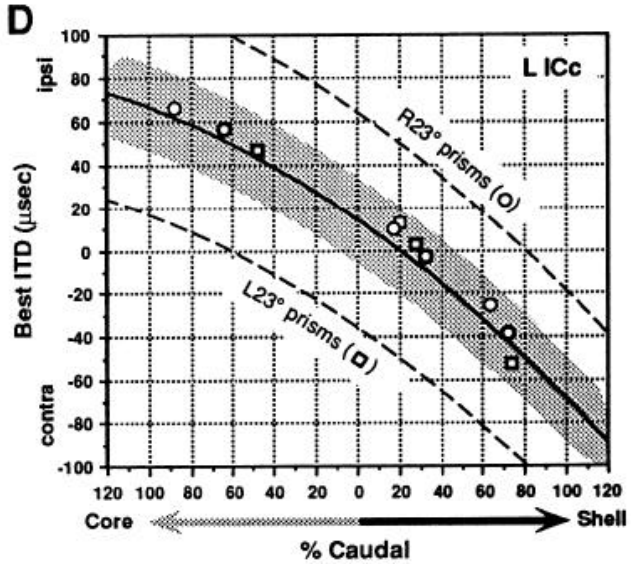

E

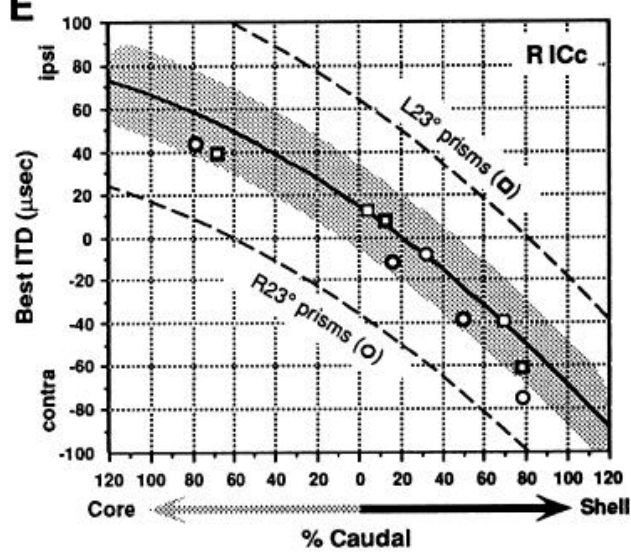

$\mathbf{F}$

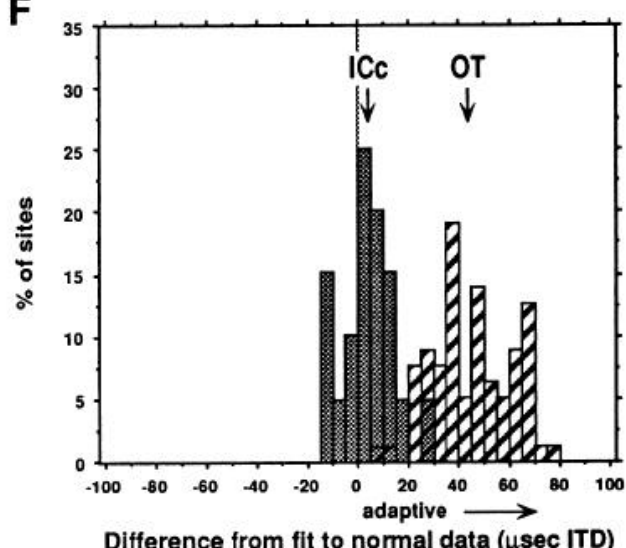

Figure 9. Representation of ITD in the ICc of normal and prism-reared owls. A, Schematic diagram of a horizontal section through the core and lateral shell of the right ICc showing how the representation of ITD in these nuclei was assessed. The locations of recording sites in anatomical reconstructions were quantified as the percentage of the distance along the rostral-caudal extents of the core (Fig. 8). The normal locations for the representation of $0 \mu \mathrm{sec}, 50 \mu \mathrm{sec}$ contra-ear-leading, and $50 \mu \mathrm{sec}$ ipsi-ear-leading ITD are indicated. The left ICc (not illustrated) is a mirror image of the right ICc. B. Normal representation of ITD in the ICc. Best ITD in the $6 \mathrm{kHz}$ lamina is plotted as a function of rostrocaudal position for 29 recording sites in the lateral shell and eight recording sites in the core from seven ICc of four normal owls. Recording sites were either determined by recovery of lesions (bold symbols) or were estimated based on their microdrive coordinates relative to recovered lesions. Values of best ITD in the lateral shell and core are plotted on the right and left half of the graph, respectively. The solid line is a second-order polynomial fit to the data and the shaded region indicates best ITD values within $20 \mu \mathrm{sec}$ of this fit. $C$, Histogram of the differences between the measured values of best ITD and the fit plotted in $B$. D and $E$, Representation of ITD in the left $(D)$ and right $(E)$ ICc of an owl raised with R23 prisms (circles, R2) and an owl raised with $\mathrm{L}_{23^{\circ}}$ prisms (squares, L2). Data are plotted in the same format as in $B$. The normal relationship between best ITD and location (solid line superimposed from $B$ ) and the relationships predicted if the representation of ITD were shifted by $50 \mu$ sec in the adaptive direction are indicated for comparison. $F$, Histogram (shaded bars) of differences between measured values of best ITD for owls R2 and L2 and values predicted from the normal relationship between best ITD and location in the ICc. Differences were assigned a positive value if they were in the adaptive direction (appropriate to account for the shift in the representation of ITD in the optic tectum). The adaptive shifts in ITD for recording sites in the optic tectum of owls R2 and L2 are plotted for comparison (hatched bars). Downward arrows indicate median values for the ICc and optic tectum $(O T)$. 
A

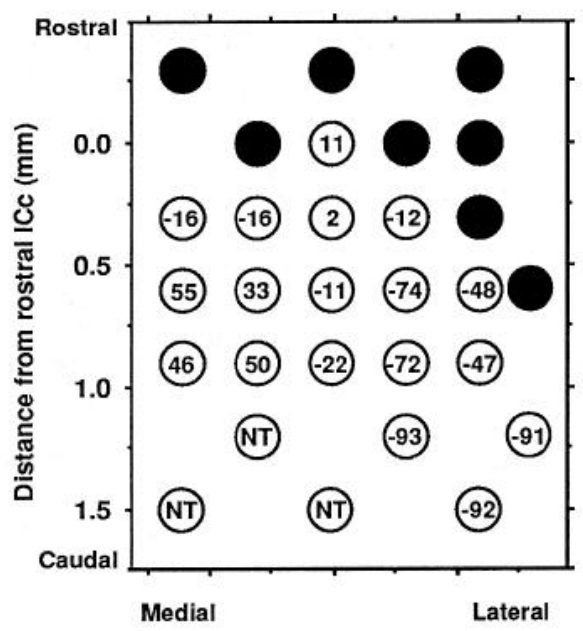

C

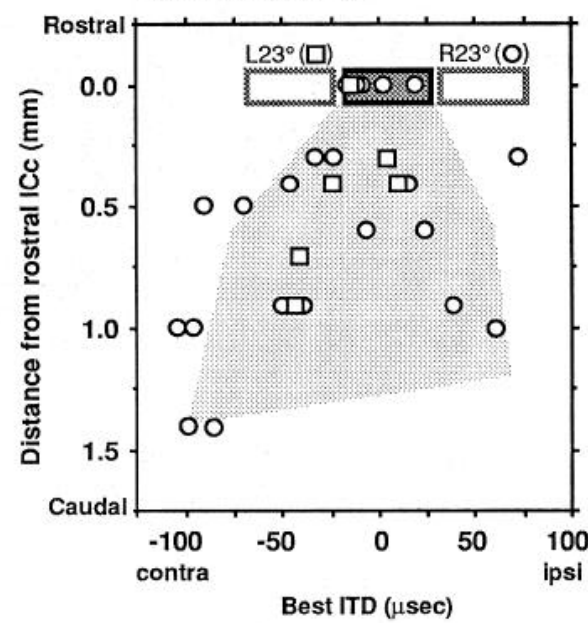

B
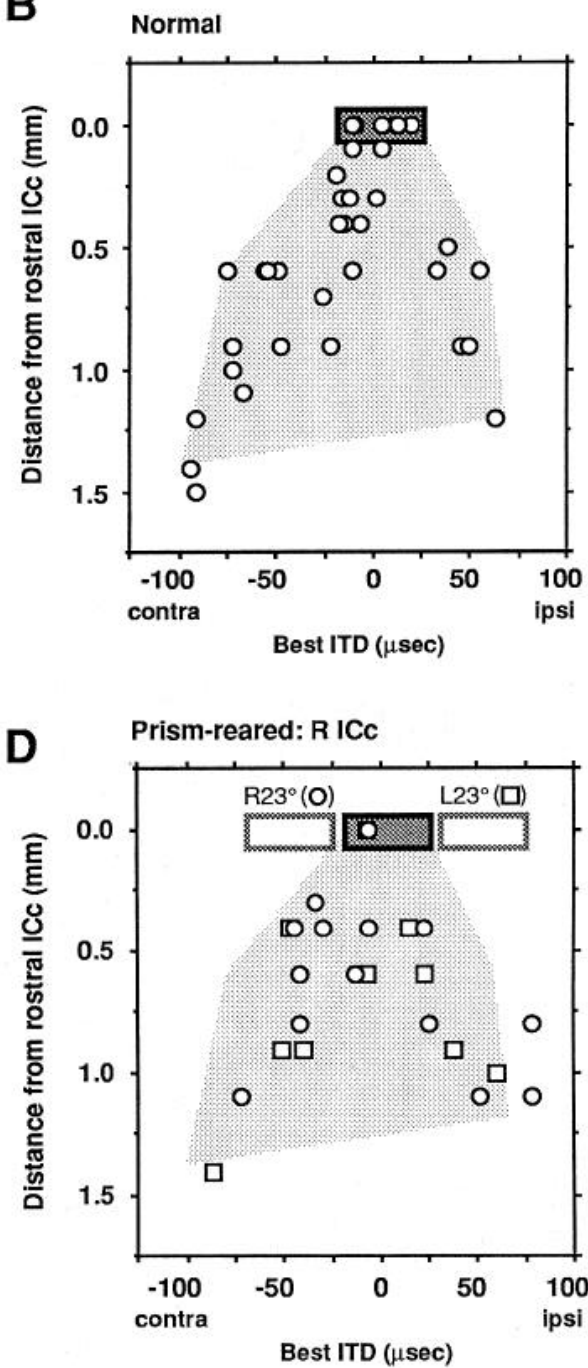

Figure 10. Best ITD values at the rostral end of the ICc in normal and prism-reared owls. A, An example from a normal owl illustrating how the representation of ITD in the ICc was assessed. Circles indicate the relative locations of a series of electrode penetrations through the rostrolateral corner of the right ICc. The physiological properties of units recorded in each penetration (see Materials and Methods) were used to assign it to either the ICx (circled Xs) or the ICc (circled numbers). Two penetrations (solid circles) passed through fibers lying rostromedial to the ICc. The values of ITD represented in the $6 \mathrm{kHz}$ lamina of the ICc were measured and are indicated for each penetration by positive numbers for ipsi-earleading ITDs and negative numbers for contra-ear-leading ITDs. For some penetrations, units were not tuned for ITD $(N T)$. $B$, Normal representation of ITD at the rostral end of the ICc. For four experiments like the one illustrated in $A$, best ITD is plotted as a function of distance from the rostralmost penetration through the ICc. The region of light shading indicates approximately the envelope of the normal data. The values of ITD measured in the rostralmost penetrations (shaded rectangle) ranged from $22 \mu \mathrm{sec}$ ipsi-ear-leading to $12 \mu \mathrm{sec}$ contra-ear-leading. $C$ and $D$, Representation of ITD at the rostral end of the left $(C)$ and right $(D)$ ICc of an owl reared with $\mathrm{R} 23^{\circ}$ prisms (circles, R1) and an owl reared with L23 ${ }^{\circ}$ prisms (squares, L1). Data are in the same format as in $B$. The values of best ITD at the rostralmost sites in the ICc of the prism-reared owls fell mostly within the normal range of best ITDs for this location (shaded rectangle, superimposed from $B$ ), and not within the ranges predicted if the representation of ITD at the rostral end of the ICc were shifted in the adaptive direction by $50 \mu \mathrm{sec}$ (open rectangles).

rostralmost locations in the ICc ranged from $12 \mu \mathrm{sec}$ contraear-leading to $22 \mu \mathrm{sec}$ ipsi-ear-leading, with a median value of $5 \mu$ sec ipsi-ear-leading (shaded rectangle, Fig. 10B). These values correspond closely to the values of best ITD determined in the lesion experiments to lie at the rostral end of the ICc (Fig. 9B).

In four ICc from two prism-reared owls, best ITD values at the rostralmost locations were within the normal range (Fig. $10 C, D$ ): best ITDs ranged from $6 \mu \mathrm{sec}$ contra-ear-leading to 15 $\mu \mathrm{sec}$ contra-ear-leading for owl $\mathrm{L} 1$, and from $16 \mu \mathrm{sec}$ contraear-leading to $20 \mu \mathrm{sec}$ ipsi-ear-leading for owl R1. Moreover, there was no tendency for the values of best ITD represented at the rostral end of the ICc to be shifted in the adaptive direction (open rectangles, Fig. 10C,D): the median shift in best ITD for the sites from the rostral ICc of prism-reared owls was $7 \mu \mathrm{sec}$ in the nonadaptive direction relative to the median value from the normal owls ( $5 \mu \mathrm{sec}$ ipsi-ear-leading).

Several best ITD values recorded from owl R1 were outside of the envelope of values that were recorded from normal owls (circles outside the region of shading, Fig. 10C,D). However, among the six best ITD values that fell outside of the normal envelope, only one was displaced in an adaptive direction. These data therefore do not argue in favor of an adaptive shift in the ICc. The data could be explained if the rostral end of the ICc was farther rostral than estimated. 
Table 2. Core staining in normal and prism-reared owls

\begin{tabular}{|c|c|c|c|c|}
\hline & \multirow[b]{2}{*}{ Owl } & \multirow[b]{2}{*}{ Side } & \multicolumn{2}{|c|}{ Rostrocaudal } \\
\hline & & & $\begin{array}{l}\text { Midpoint } \\
(\%)^{a}\end{array}$ & Length $(\%)^{a}$ \\
\hline \multirow[t]{7}{*}{ Normal } & \multirow[t]{2}{*}{1} & $\mathbf{R}$ & 43 & 34 \\
\hline & & L & 45 & 29 \\
\hline & \multirow[t]{2}{*}{2} & $\mathbf{R}$ & 44 & 30 \\
\hline & & L & 38 & 38 \\
\hline & \multirow[t]{2}{*}{3} & $\mathbf{R}$ & 43 & 28 \\
\hline & & $\mathbf{L}$ & 43 & 38 \\
\hline & \multirow[t]{2}{*}{4} & \multirow[t]{2}{*}{$\mathbf{R}$} & 46 & $\underline{38}$ \\
\hline Normal average & & & $\overline{43.1}$ & $\overline{33.6}$ \\
\hline \multirow[t]{2}{*}{$\mathrm{L} 23^{\circ}$ prisms } & \multirow[t]{2}{*}{$\mathrm{L} 2$} & $\mathbf{R}$ & 42 & 33 \\
\hline & & L & 45 & 33 \\
\hline \multirow[t]{2}{*}{$\mathrm{R} 23^{\circ}$ prisms } & \multirow[t]{2}{*}{$\mathrm{R} 2$} & $\mathbf{R}$ & 44 & 37 \\
\hline & & L & 42 & 35 \\
\hline Prism average & & & $\overline{43.3}$ & 34.5 \\
\hline
\end{tabular}

${ }^{a}$ Measured at the dorsoventral level of the $6 \mathrm{kHz}$ lamina and expressed as percentage of the rostrocaudal extent of the ICx.

The data from both the lesion and mapping studies indicate that, in prism-reared owls, the representation of ITD in the ICc is normal and cannot account for the shift in the representation of ITD at the level of the optic tectum. We therefore investigated the representation of ITD in the ICx, which lies between the ICc and the optic tectum in the ascending ITD pathway (Fig. $1)$.

\section{The external nucleus of the inferior colliculus}

The results presented in the previous sections indicated that the visual map of space in the optic tectum and the map of ITD in the ICc lateral shell were not altered by prism rearing. Therefore, these maps were used as physiological landmarks to specify locations of recording sites in the ICx. For six ICx from six normal owls and six ICx from three prism-reared owls, ITD tuning was measured in a series of dorsoventral penetrations along a transect between the representation of $0 \mu \mathrm{sec}$ ITD in the ICc lateral shell and the representation of $0^{\circ}$ azimuth in the visual map of space in the optic tectum (Fig. 11A). For all transects, the medialmost penetrations passed through the $6 \mathrm{kHz}$ lamina of the lateral shell at locations where the best ITD was within $12 \mu \mathrm{sec}$ of 0 ITD. The lateralmost penetrations entered the optic tectum at locations where VRFs were within $3^{\circ}$ of the midsagittal plane and less than $10^{\circ}$ above the horizon. The border between the ICx and the optic tectum was indicated by a region approximately $60 \%$ of the way from the ICc to the lateral portion of the optic tectum, where there was little or no spontaneous or stimulus-driven activity, corresponding to the region in and around the tectal ventricle. The border between the ICc and ICx was defined physiologically (see Materials and Methods).

In normal owls, the ITD values represented along this transect were close to $0 \mu \mathrm{sec}$. Figure $11 B$ shows the median best ITD for each penetration as a function of the location of the penetration along the transect. Median best ITDs at all locations in the ICx (vertical shaded region, Fig. $11 B$ ) and optic tectum were within $10 \mu \mathrm{sec}$ of 0 ITD (horizontal shaded region, Fig. 11B). The transect therefore passes through the auditory representation of frontal space in the ICc, ICx, and optic tectum. This
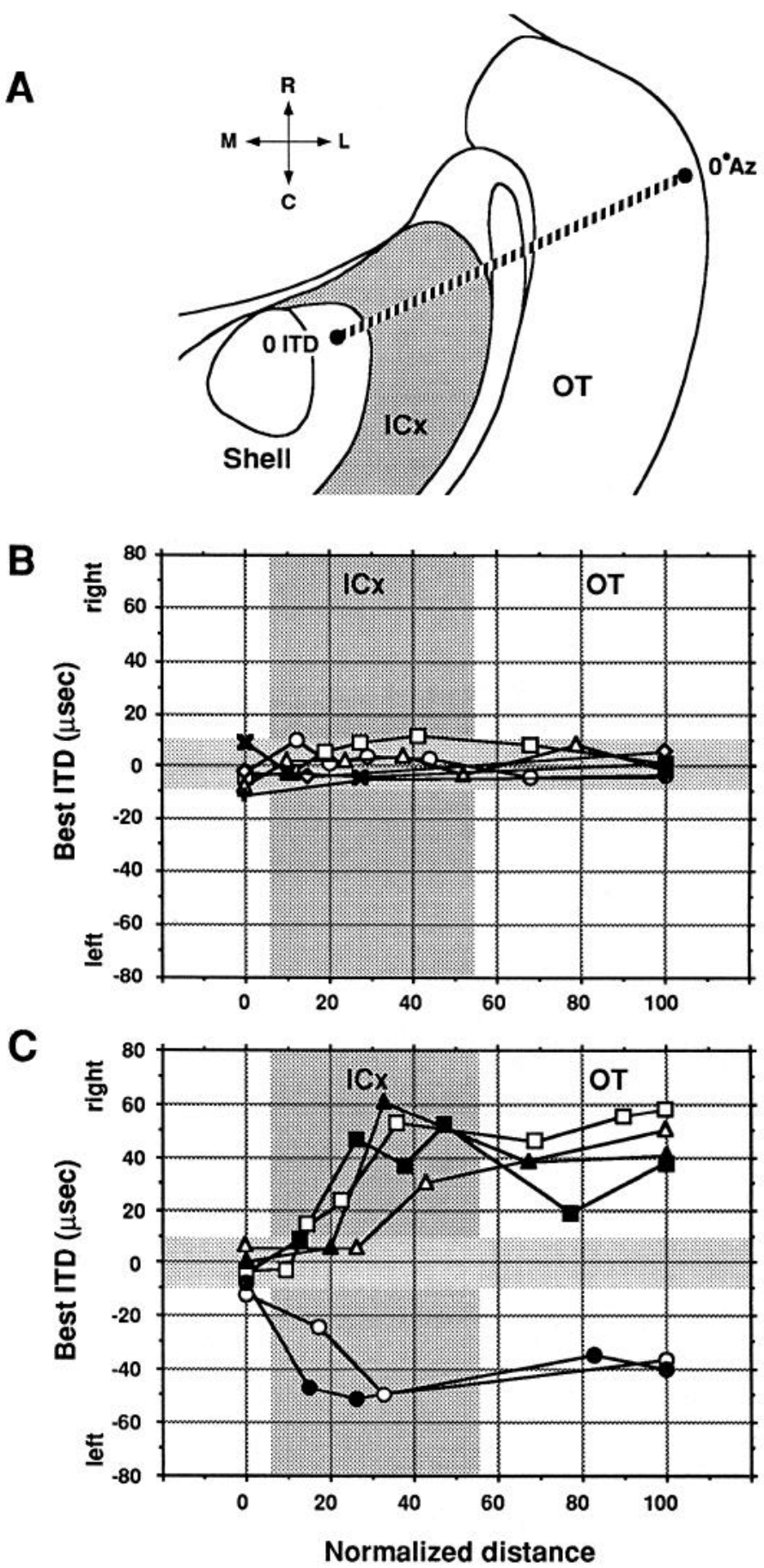

Figure 11. Representation of ITD in the ICx of normal and prismreared owls. $A$, Schematic diagram of a horizontal section through the ICc, ICx, and optic tectum (OT) illustrating the transect (hatched line) along which the representation of ITD in the ICx was assessed. $B$, Summary of best ITDs measured along transects across six ICx in six normal owls. Each data point represents the median value of best ITD for all recording sites in a penetration through the ICx (dark shading) or optic tectum. Best ITD is plotted as a function of normalized distance along the transect. Solid lines connect data from individual experiments. The median best ITDs for all penetrations across the ICx and optic tectum were within $10 \mu \mathrm{sec}$ of 0 ITD (light shading). $C$, Summary of best ITDs measured along transects across three right ICx (open symbols) and three left ICx (solid symbols) in three prism-reared owls (squares, L1; triangles, L2; circles, R2). The normal range of ITDs (light shading) is superimposed from $B$. 

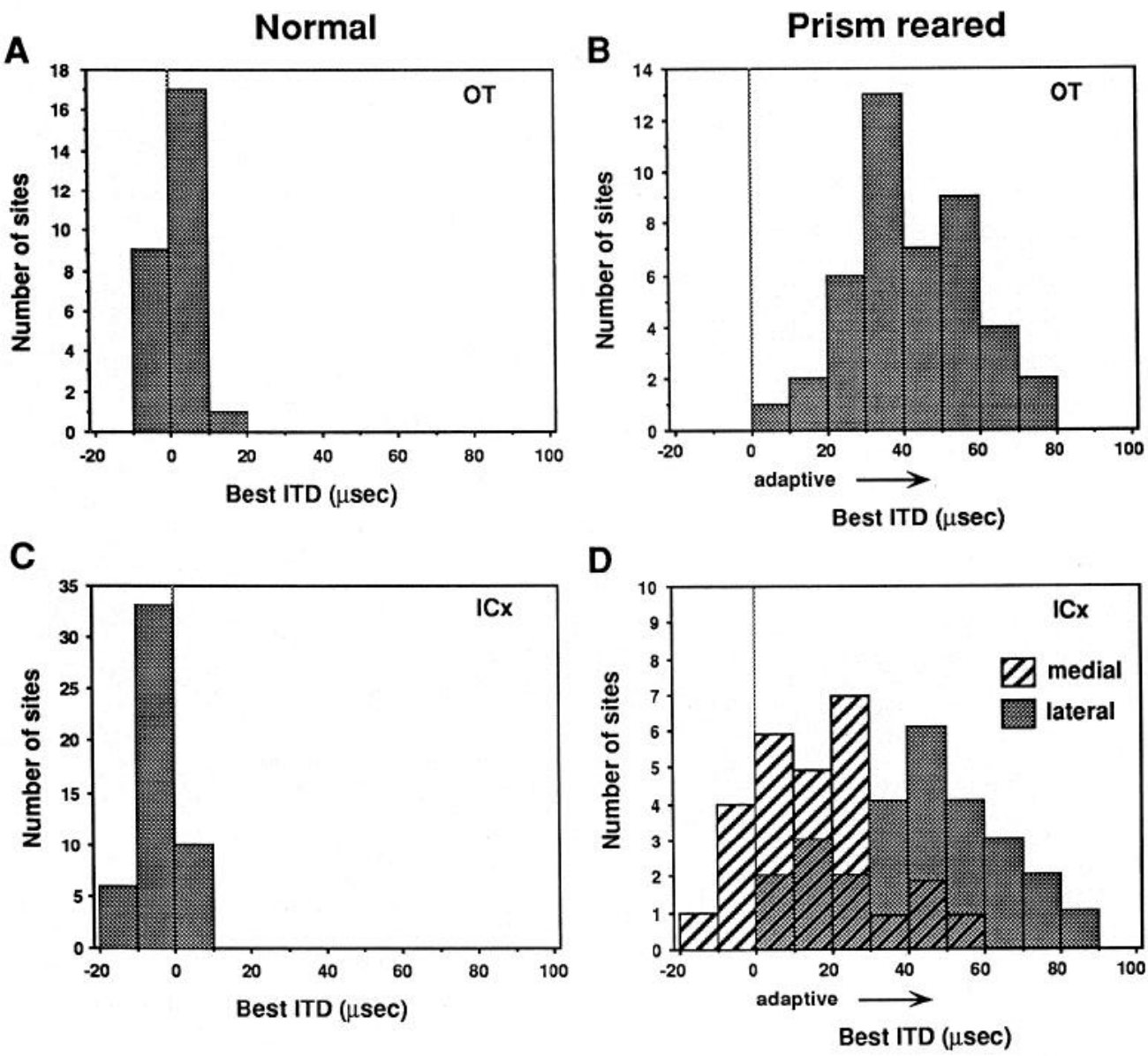

Figure 12. Best ITD values for all recording sites in the ICx and optic tectum of normal and prism-reared owls along the transects shown in Figure 11. $A$, Best ITDs for sites in the optic tectum of normal owls. The median best ITD was $2 \mu \mathrm{sec}$ ipsi-ear-leading. $B$, Best ITDs for sites in the optic tectum of prism-reared owls. Best ITDs were assigned positive values if they were shifted in the adaptive direction relative to $0 \mu \mathrm{sec}$ ITD. $C$, Best ITDs for sites in the ICx of normal owls. The median best ITD was $4 \mu \mathrm{sec}$ contra-ear-leading. $D$, Best ITDs for sites in the medial half (hatched bars) and lateral half (shaded bars) of the ICx of prism-reared owls.

corresponds to a region of the visual field that was displaced in all the prism-reared owls.

In the optic tectum of the prism-reared owls, the representation of ITD was shifted (Fig. 11C), as expected from prior results (Fig. 5). In the ICx, the representation of ITD was also shifted, in a direction appropriate to account for the shift observed in the optic tectum (Fig. 11C). There was an apparent increase in the amount of shift across the mediolateral extent of the ICx: at locations close to the physiologically defined border between the ICc and ICx there were relatively small shifts in the representation of ITD, while in the lateral portion of the ICx, the representations of ITD were shifted outside of the normal range by amounts approximately equal to the shifts measured in the optic tectum. The magnitude of the shifts was quantified as the difference between measured ITD tuning and the expected normal tuning $(0 \mu \mathrm{sec})$ for recording sites in the optic tectum, the medial $50 \%$ of the ICx, and the lateral $50 \%$ of the ICx (Fig. 12). The median adaptive shift for the optic tectum was $40 \mu \mathrm{sec}$ (Fig. 12B). For the medial half of the ICx (hatched bars in Fig. 12D), there was only a small adaptive shift in best ITD, with a median value of $10 \mu \mathrm{sec}$ in the adaptive direction. For the lateral half of the ICx (shaded bars in Fig. $12 D)$, the median shift in best ITD was $47 \mu \mathrm{sec}$. This shift was large enough to account for the shift measured in the optic tectum of the same owls (Fig. 12B).

To determine the magnitude of the displacement of the ITD map across the ICx, lesions were placed at critical locations in eight ICx of five normal and in five ICx of four prism-reared owls (Fig. 13). Lesions were placed in the lateralmost part of the ICx (Fig. 13A-C), where the shift in the representation of ITD was largest. The locations of the lesions were quantified in horizontal Nissl-stained sections, as the distance from the rostral end of the ICx to the center of the lesion divided by the distance from the rostral to the caudal pole of the ICx (Fig. 13D). In normal owls, best ITDs varied systematically with rostrocaudal location in the ICx (open circles in Fig. 13E). Best ITDs close to $0 \mu \mathrm{sec}$ were represented at locations approximately $20 \%$ caudal in the ICx, and best ITDs of $50 \mu \mathrm{sec}$ contra-ear-leading were represented at locations approximately $50 \%$ caudal. In the prismreared owls, values of ITD were represented at locations that were displaced from normal in a direction that was appropriate to account for the shift in the representation of ITD in the optic tectum (Fig. 13E). On the right side of two owls reared with $\mathrm{R} 23^{\circ}$ prisms (solid circles) and the left side of an owl reared with $\mathrm{L}_{23^{\circ}}$ prisms (solid square) contralateral best ITDs were measured at locations in the ICx that were approximately $40 \%$ more rostral than normal. On the left side of two owls reared with $\mathrm{R} 23^{\circ}$ prisms (solid triangles) ipsilateral best ITDs were also represented at abnormal locations. The data indicate that the representations of ITD in the ICx had shifted in the adaptive direction by approximately $1.5 \mathrm{~mm}$.

Because the amount of shift in the representation of ITD appeared to vary with mediolateral location in the ICx, we examined whether other unit properties also varied. Unit tuning for ITD and ILD normally sharpens, and unit tuning for frequency broadens, along the auditory pathway leading to the optic tectum. For both normal and prism-reared owls, these properties did not differ significantly between recording sites in 

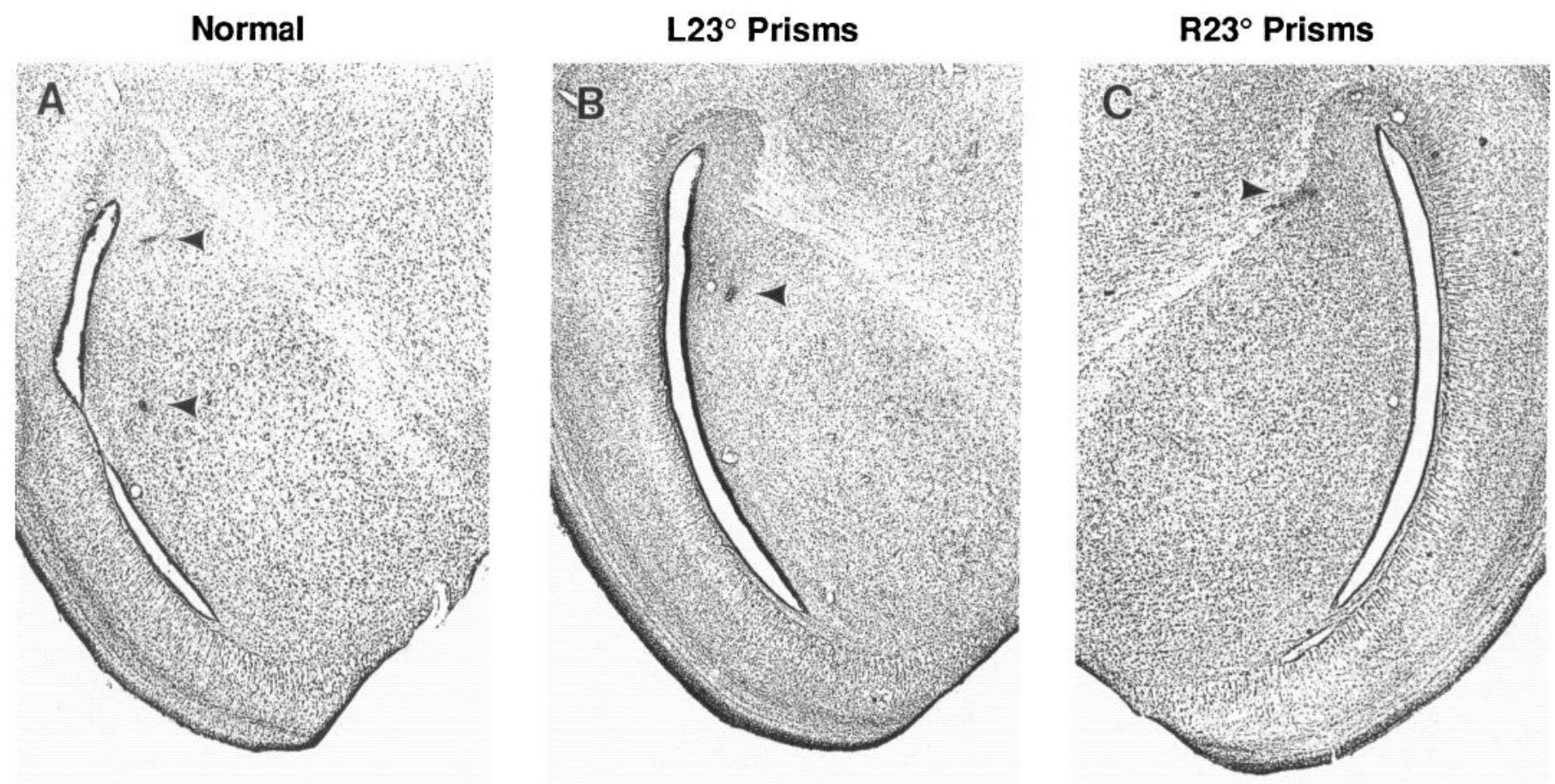

$1 \mathrm{~mm}$
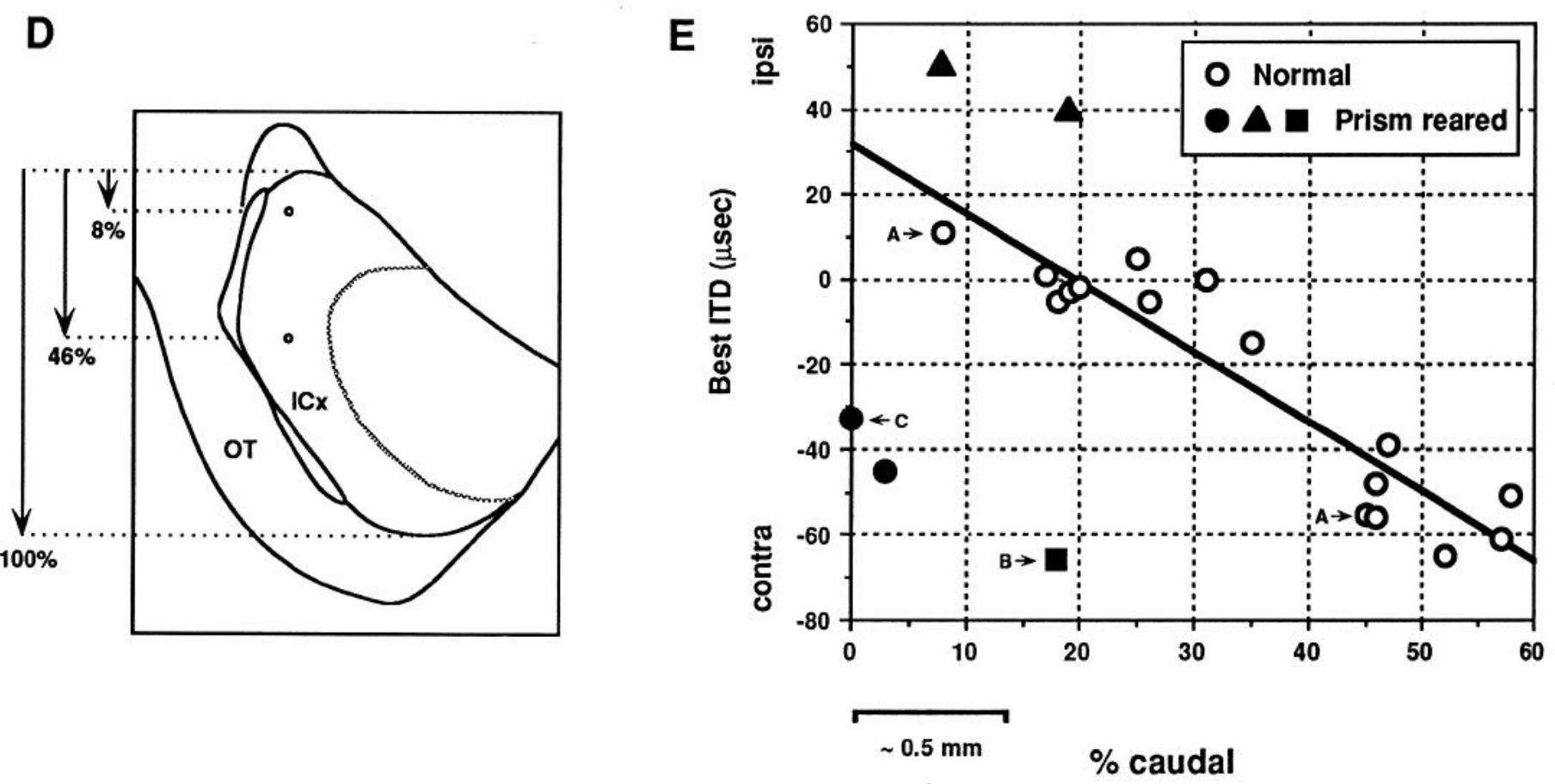

Figure 13. Best ITD as a function of anatomical location in the ICx of normal and prism-reared owls. $A-C$, Nissl-stained, horizontal sections through the ICx of normal and prism-reared owls showing recording sites, marked by lesions (arrowheads), at which best ITDs were measured. $D$, Camera lucida drawing of the section shown in $A$ : location was quantified as the percentage of distance from the rostral to the caudal pole of the ICx. E, Plot of best ITD as a function of rostrocaudal location in the ICx for normal owls (open symbols) and prism-reared owls (solid symbols). The solid line is a linear least-squares fit to the normal data. Letters indicate data points corresponding to the lesions in $A-C$.

the medial and lateral portions of the ICx. Latencies of responses to optimal auditory stimuli, however, were significantly longer for sites in the lateral ICx than for sites in the medial ICx (Fig. 14A). This was particularly true for prism-reared owls, where several units with exceptionally long latencies were recorded in the lateral part of the ICx.
The sites with the longest latencies in the ICx of prism-reared owls were also among the sites with the largest shifts in best ITD (Fig. 14B). Many large shifts in best ITD were also measured among the sites with the shortest latencies. However, best ITD measurements for these sites were based on the spikes collected during the $100 \mathrm{msec}$ following stimulus onset, and 

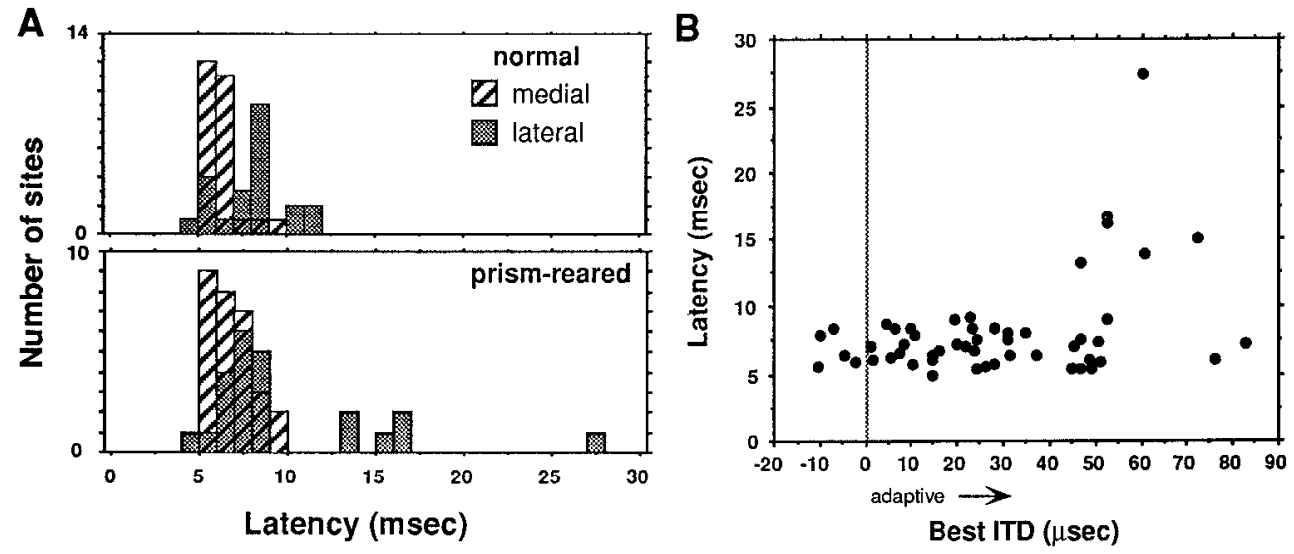

Figure 14. Latencies of unit responses recorded in the ICx of normal and prism-reared owls. $A$, Histograms of latencies at sites from the ICx of normal (top) and prism-reared (bottom) owls. Data are from recording sites along the transects plotted in Figure 11. Latencies from the medial $50 \%$ of the transect across the ICx (hatched bars) were significantly shorter $(p<0.01)$ than those for more lateral sites in the ICX (shaded bars) in both normal and prism-reared owls. $B$, Latency is plotted as a function of adaptive shift in best ITD for sites from the ICx of prism-reared owls. therefore did not reveal whether the earliest components of the responses were tuned to shifted best ITDs. If only the later portions of the responses were tuned to shifted values of ITD, then it would be possible that shifted best ITDs in the ICX of prism-reared owls derived, not directly from the ICc, but from a different, longer-latency pathway, such as a descending projection from higher auditory centers.

To address this possibility, we examined the earliest responses of units in the ICx to determine the shortest latencies at which there was evidence of a shift in the representation of ITD (Fig. 15). For a few sites, ITD tuning during the initial portion of the response was less shifted than during later parts of the response (e.g., Fig. 15A). However, several of the sites with the largest shifts in ITD had clearly shifted ITDs within the first 7 or 8 msec following stimulus onset (e.g., Fig. 15B,C). This was the shortest interval within which ITD could be determined for any of the units from normal or prism-reared owls. Figure $15 D$ summarizes the differences in ITD tuning for early and late portions of the response. The diagonal line indicates equal values of best ITD for the early and late portions of the response. For the majority of sites, the best ITD for the first $3 \mathrm{msec}$ of the response was comparable to the best ITD determined for the entire response.

\section{Discussion}

In this study we measured the effect of laterally displaced vision on the dichotic tuning of neurons in the optic tectum and traced these changes to their point of origin in the ascending auditory pathway. We found that visual experience influenced powerfully the tectal map of ITD, controlling both the position of the map in the optic tectum and its topography. We then demonstrated that visually guided changes in the ITD map at the level of the optic tectum reflect neuronal plasticity that occurs at the next earlier level of the auditory pathway, the $\mathrm{ICx}$.

\section{Influence of vision on the dichotic tuning of neurons in the optic tectum}

Owls were reared with laterally displacing prisms with the expectation that this would primarily alter unit tuning for ITD. This was the case. The best ITDs of tectal units were adaptively shifted toward values of ITD produced by sound sources at the locations of their optically displaced VRFs (Figs. 5-7). In contrast, there was relatively little systematic influence on unit tuning for ILD. For most frequencies to which tectal neurons are responsive, stimulus ILD varies primarily with the elevation of a sound source. However, particularly for low frequencies, ILDs also vary with azimuth, from right-ear-greater to left-ear-greater as a stimulus moves from right to left (Moiseff, 1989; OIsen et al., 1989; Knudsen et al., 1991; Brainard et al., 1992). This may explain why the small shifts in unit tuning for ILD tended to be in the direction of VRF displacement.

The influences of displaced vision on dichotic tuning in the optic tectum are largely consistent with results of free-field measurements (Knudsen and Brainard, 1991). Analogous to results from free-field measurements, shifts in ITD tuning were maximal for units in the region of the optic tectum that represented the optically displaced portion of the visual field, but were also significant for surrounding regions of the optic tectum representing the portion of the visual field that was obstructed (Fig. 6). This indicates that, at least in the absence of conflicting visual guidance, changes in one portion of the auditory map may influence surrounding portions of the map. Also, as expected from free-field measurements, the representation of ITD in the optic tectum of prism-reared owls extended to more extreme values of best ITD than are normally present (e.g., Fig. 7), indicating that the ITD values actually represented in the optic tectum are selected, based on experience, from a wider possible range.

The topography of the ITD map was altered dramatically by prism rearing (Fig. 16). The magnification of ITD values (in micrometers of tissue per degree of best ITD) was quantified using the formula

$$
=\Delta \mathrm{VRF} \text { az } / \Delta \text { best ITD } \cdot \Delta \text { location } / \Delta \mathrm{VRF} \text { az, }
$$

where the first term on the right was determined from the relationship between best ITD and VRF azimuth measured in this study (Figs. 3, 5), and the second term was extracted from the published relationship between VRF azimuth and location in the optic tectum (Knudsen, 1982). For normal owls, as previously reported (Olsen et al., 1989), the magnification of ITD values near $0 \mu \mathrm{sec}$ was more than twice the magnification of ITD values greater than $50 \mu \mathrm{sec}$ ipsi- or contra-ear-leading (Fig. $16 B$ ). In contrast, the values of ITD that were magnified maximally in prism-reared owls were those that were $40-60 \mu \mathrm{sec}$ in the adaptive direction, corresponding to frontal visual fields, and the magnification of $0 \mu \mathrm{sec}$ ITD was decreased (Fig. 16B). In addition, abrupt changes in magnification occurred at locations corresponding to borders between deprived and displaced zones (e.g., the region representing approximately $-100 \mu \mathrm{sec}$ in Fig. 16). Together, these data demonstrate that vision can dictate 
A
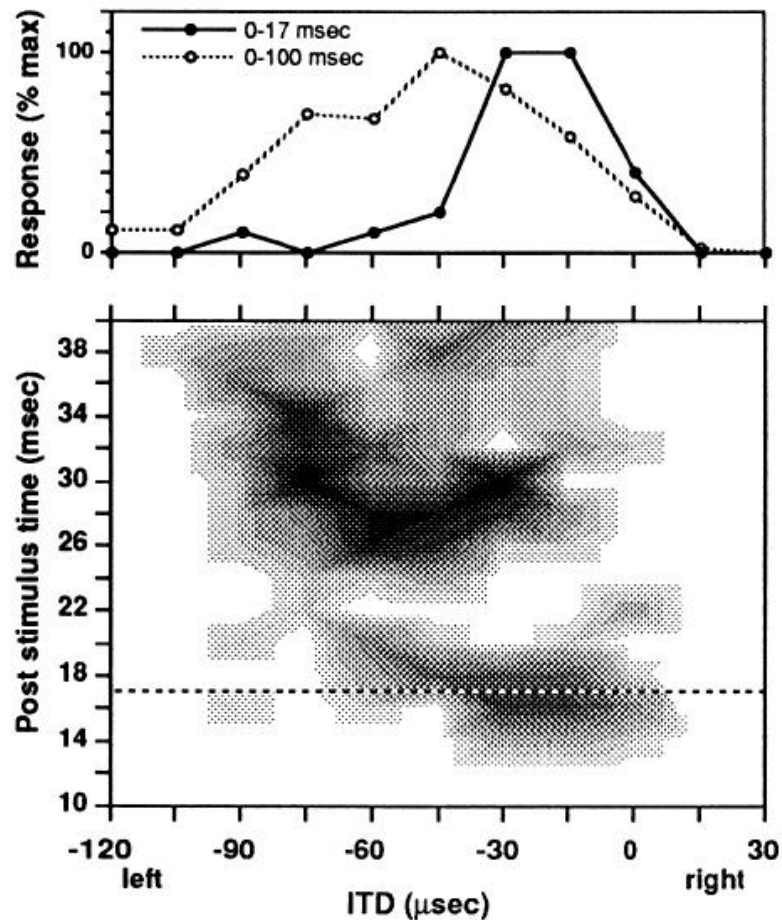

C
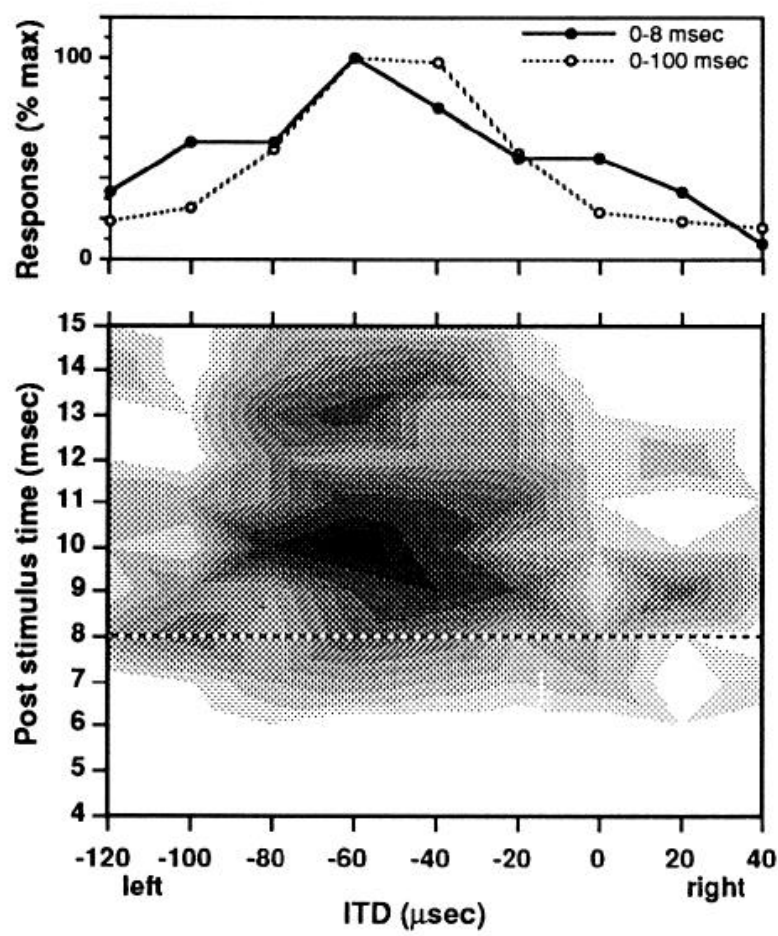

B
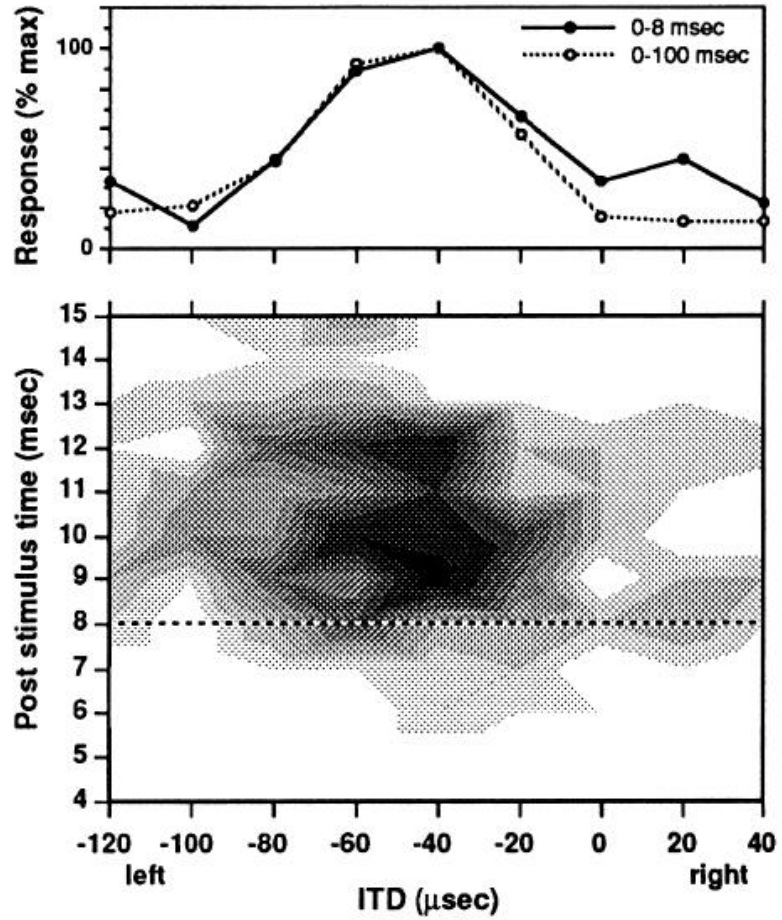

D

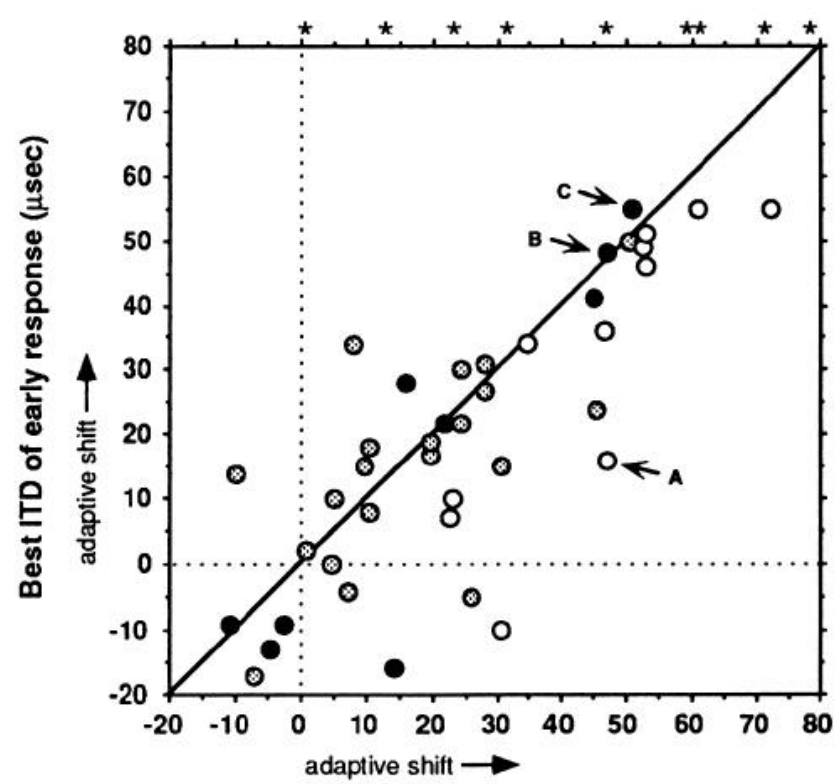

Best ITD of entire response $(\mu \mathrm{sec})$

Figure 15. ITD tuning at short latencies for units in the ICx of prism-reared owls. $A-C$, ITD tuning as a function of time for three ICx sites. Top, Responses as a function of stimulus ITD for the initial portion of the response (solid symbols) and for the entire response (open symbols). Bottom, Contour plot of response as a function of ITD and poststimulus time. For all three sites the normally expected best ITD was approximately $0 \mu$ sec, and the adaptive direction of shift was toward left-ear-leading. $A$ shows data from a site where the adaptive shift in ITD tuning was small early in the response and increased with time. $B$ and $C$ show data from sites that had short latencies $(5-6 \mathrm{msec})$ and large adaptive shifts in ITD tuning from the onset of their responses. $D$. Adaptive shifts in ITD determined for the first $3 \mathrm{msec}$ of response are plotted against shifts determined for the entire $100 \mathrm{msec}$ following stimulus onset for recording sites from transects across the ICx of prism-reared owls (Fig. 11C). Data are indicated separately for sites at which ITD tuning could be determined within $8 \mathrm{msec}$ of stimulus onset (solid circles), within 8-10 msec of stimulus onset (shaded circles), or more than $10 \mathrm{msec}$ following stimulus onset (open circles). The letters indicate points corresponding to the plots in $A-C$. Points above the graph indicate sites for which ITD tuning could not be determined during the first $3 \mathrm{msec}$ of response. The solid line indicates equivalent shifts for the initial response and for the entire response. Many sites had shifted best ITDs from the onset of responses. 
A

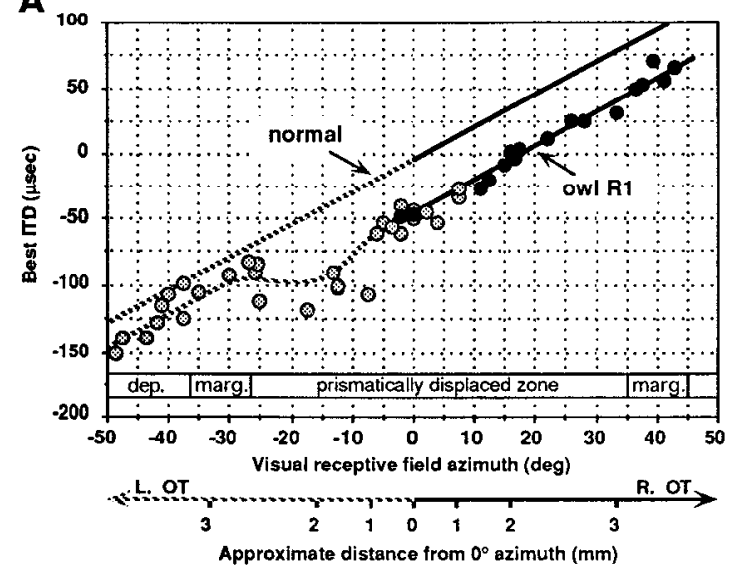

B

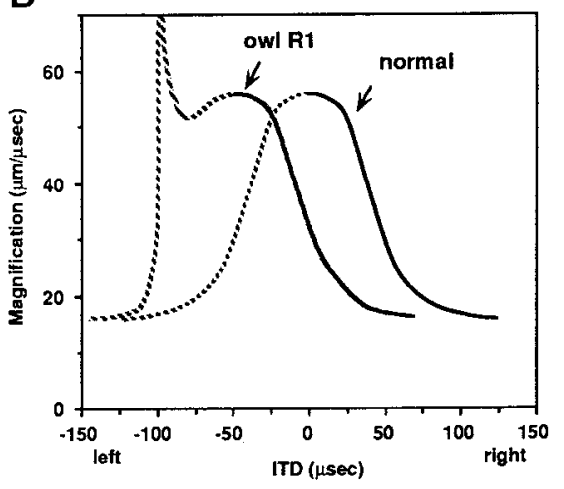

Figure 16. Abnormal topography of the ITD map in a prism-reared owl. A, Best ITD is plotted as a function of VRF azimuth for units from the right optic tectum (shaded symbols) and left optic tectum (solid symbols) of an owl raised with $\mathrm{R}_{23}^{\circ}$ prisms (R1). Also indicated (at bottom) is the approximate location in the optic tectum at which each VRF azimuth is represented (from Knudsen, 1982). The straight line indicates the normal relationship between best ITD and VRF azimuth (replotted from Fig. $3 C$ ). The curved line through the data points was fit by eye. The amount of shift in the representation of ITD was approximately constant for units with VRFs in the prismatically displaced zone and decreased abruptly for units with VRFs at the left border between the deprived and marginal zones. $B$, Magnification as a function of ITD for the right optic tectum (hatched lines) and left optic tectum (solid lines) of normal owls and owl R1. Magnification was calculated from the relationship between best ITD and VRF azimuth plotted in $A$, and the relationship between VRF azimuth and location in the optic tectum (from Knudsen, 1982).

the magnification, and therefore the topography, of the tectal map of ITD.

Central representations of visual, somatosensory, and auditory information have been shown to be altercd by differential activity in the sensory periphery, with increased or decreased activity leading to expansion or contraction, respectively, of the corresponding portion of the sensory map (visual: Hubel et al., 1977; Shatz and Stryker, 1978; LeVay et al., 1980; Kaas et al., 1990; Heinen and Skavenski, 1991; Gilbert and Wiesel, 1992; somatosensory: Merzenich et al., 1984; Jenkins et al., 1990; auditory: Robertson and Irvine, 1989). These examples suggest the importance of activity-dependent competition in establishing the topography of neural maps.

In contrast, the dramatic changes in the topography of the auditory space map described here occur despite essentially normal auditory experience. This example indicates that the topography of a sensory map can be actively guided by information provided through another sensory source. This process has its closest parallel in the development of ipsilateral and contralateral eye maps in the optic tectum of Xenopus: if onc eye is rotated, disrupting the normal alignment between left and right eye visual maps, the indirect projection from the ipsilateral eye reorganizes to bring the two maps back into alignment (Gaze et al., 1970; Udin and Keating, 1981). Both in the barn owl and in Xenopus, the topography of one map (auditory or visual) actively conforms to the topography of the visual map from the contralateral eye.

\section{Site of plasticity}

The results demonstrate that the ICx is the lowest level in the ITD pathway to the optic tectum where visual experience alters the representation of auditory space. In the ICc lateral shell, which provides input to the ICx, the representation of ITD was unaffected by prism rearing (Figs. 9, 10). However, in the ICx the representation of ITD was shifted by an amount and in a direction that matched the shift measured in the optic tectum for the same owls (Figs. 11-13). Thus, plasticity in the repre- sentation of ITD in the ICx could completely account for the visually guided changes in the representation of ITD in the optic tectum.

The ICx is the first site in the ascending pathway to the optic tectum where sound localization cues are combined to create units with single, restricted auditory receptive fields, and where units are organized to form a systematic representation of auditory space. This representation is conveyed by a topographic projection to the optic tectum (Knudsen and Knudsen, 1983), where it is combined with spatial representations from other sensory modalities. It seems likely, therefore, that the ICx, at least in owls, is specialized for processing explicitly spatial aspects of auditory stimuli. In contrast, the ICc probably participates in all aspects of auditory processing; it lies on the main ascending auditory pathway to the forebrain, providing a major source of input to the auditory thalamus. Thus, prism rearing, which changes the spatial meaning of auditory cues but does not alter the cues themselves, causes adaptive changes only in the portions of the ascending auditory pathway that are specifically concerned with interpreting auditory spatial cues.

Across the mediolateral extent of the ICx there appeared to be an increase in the amount of prism-induced shift in the representation of ITD (Figs. 11, 12). Units recorded within about $750 \mu \mathrm{m}$ of the physiologically defined border between the ICc lateral shell and the ICx tended to have small adaptive shifts, while units farther lateral had shifts as large as those observed in the optic tectum. The significance of this is not clear. Although it is possible that some of the "ICx" sites with small ITD shifts were actually miscategorized ICc sites, the majority of sites with small ITD shifts were indistinguishable, based on tuning to frequency and ILD, from ICx sites with larger shifts. It is also possible that the progression of ITD shifts near the ICc/ICx border is an artifact of multiunit recording: intermediate ITD shifts potentially could result from a nonselective electrode recording from a combination of units with unshifted ITD tuning in the ICc (or fibers projecting from the ICc to the ICx) and units with shifted ITD tuning in the ICx. This possibility is 
unlikely, however, because we did not observe broader ITD tuning curves for units with intermediate values of shift (as might be expected from the addition of shifted and unshifted ITD tuning curves). Even if this had been the case, it could not explain the presence of completely unshifted ITD tuning curves at sites that had ICx-like properties.

Differences in the amount of shift across the mediolateral extent of the ICx could reflect a serial processing of ITD across as yet unidentified subnuclei or layers of the ICx. There is, however, little physiological or anatomical data that support such divisions. Indeed, retrograde tracing studies in the barn owl indicate that cells along the entire mediolateral extent of the ICx project in parallel to the optic tectum (Knudsen and Knudsen, 1983; Knudsen, 1985). Furthermore, in this study, there was only a small increase in latency at increasingly lateral sites in ICx, and large shifts in best ITD were found among the ICx units with the shortest latencies (Figs. 14, 15). An alternative explanation for the differences in the amount of shift in the medial and lateral $\mathrm{ICx}$ could be the presence of a stronger visually based instructive signal in the lateral part of the ICx or a greater capacity of the connections from the ICc to the lateral part of the ICx to respond to such a signal. Distinguishing between these alternatives will require studies of the connectivity between the ICc and the ICx and of the microcircuitry of the ICx.

Anatomical and physiological evidence strongly supports the hypothesis that the auditory map of space in the ICx derives from ascending auditory input from the ICc (Knudsen and Knudsen, 1983; Takahashi and Konishi, 1986; Wagner et al., 1987), and therefore that the visually guided shift in the ITD representation at the level of the ICx reflects plasticity in this pathway. However, in many vertebrate species the $\mathrm{ICx}$ receives a descending projection from auditory cortex (for a review, see Huffman and Henson, 1990), and for a few species a projection from the cerebellum has been reported (Carpenter et al., 1958; Carpenter, 1959; Earle and Matzke, 1974; Zimminy et al., 1981). In the barn owl, space-tuned auditory neurons have been recorded in the vermis of the cerebellum (Norris, 1982) and in field L of the forebrain (Knudsen et al., 1977). In principle, projections (that have not been documented for the barn owl) from these structures could contribute to the representation of auditory space in the ICx, and plasticity in these pathways could be responsible for the visually guided shift in the representation of ITD in the ICx. However, the relative latencies of auditory responses in the cerebellum, forebrain, and $\mathrm{ICX}$ argue against this possibility. Many ICx units with large adaptive shifts in ITD had latencies as short as $5-6 \mathrm{msec}$ (Fig. 14) and were tuned to shifted values of ITD from the onset of their responses (Fig. 15). In the vermis of the barn owl's cerebellum, the shortest latencies of space-tuned neurons are also 5-6 msec (Norris, 1982), and since activity in the vermis would have to pass through at least one synapse in the deep cerebellar nucleus before reaching the $\mathrm{ICx}$, it is unlikely that cerebellar input drives the early auditory responses in the ICx. The earliest auditory responses in the forebrain occur even later, with latencies of 9 $10 \mathrm{msec}$ ( $\mathrm{Y}$. Cohen, personal communication), and are even less likely to contribute to early responses in the $\mathrm{ICx}$. Therefore, at least the short-latency responses to shifted values of ITD reflect plasticity in the ascending auditory pathway at the level of the ICx.

Although the ICx is a site of plasticity, it is probably not the only one. As mentioned above, there is evidence for neural representations of auditory space in the cerebellum and forebrain, and behavioral experiments indicate that at least forebrain and tectal pathways contribute in parallel to sound localization (Knudsen and Knudsen, 1993). The connectivity between different auditory representations in the brain and their relative contributions to sound localization behavior are not well understood. If a representation of auditory space is created only at the level of the ICx and then conveyed to other regions of the brain where auditory space is represented (such as the optic tectum, forebrain, and cerebellum), then the ICx might indeed be the only site of plasticity. However, if representations of auditory space are derived at several different sites, it seems likely that visually guided adjustments at all levels would occur in parallel so that the representations remain mutually consistent.

\section{Potential sources of an instructive signal}

The data indicate that information provided by vision can influence the representation of auditory space in the ICx. How might this occur? One purely sensory hypothesis is that there is a retinotopic map at the site of visual instruction that serves as a spatial template for the auditory map: a bimodal (auditoryvisual) environmental stimulus could activate a point in the visual map and, via a Hebbian mechanism (Hebb, 1949; Brown et al., 1990), cause a strengthening of concurrently active auditory inputs. Such mechanisms, involving the selection of sensory inputs that are activated concurrently with an instructive signal, have been demonstrated directly in visual cortex (Fregnac et al., 1988, 1992; Gruel et al., 1988; Shulz and Fregnac, 1992) and implicated in other examples of sensory plasticity (Udin and Fawcett, 1988). A second, sensory-motor hypothesis is that vision provides feedback on the accuracy of the auditory localization of bimodal stimuli: orienting head movements based on auditory cues could be followed by a visual assessment of the discrepancy between the direction of gaze and the location of the stimulus, and this "error signal" could be used to adjust the auditory map. Such a visually based motor error signal provides the basis for the calibration of the vestibulo-ocular reflex (Lisberger, 1988). Our results do not directly address which, if either, of these mechanisms is responsible for the influence of vision on the representation of auditory space.

Whatever the nature of the visually based instructive signal, how does it reach the $\mathrm{ICx}$ ? The only documented source of input to the ICX in barn owls is the ICc, generally considered to be a purely auditory nucleus. However, as mentioned above, projections from both the forebrain and the cerebellum to the ICX have been reported in some species. Although the nature of the signals conveyed by these projections is not well characterized, they are potential sources of an instructive signal to reorganize the representation of ITD in the ICx. Also, in several species, a sparse retina-to-ICx projection has been demonstrated (Itaya and Hoesen, 1982; Yamauchi and Yamadori, 1982; Jen et al., 1984; Kunzle, 1988). Such a projection in owls could provide a visually based spatial template to which the auditory map of space is matched. However, there is no evidence that this projection is topographic or that it forms functional synapses in the ICx, and visual responses have not been recorded in the ICx of any species.

The experiments in this study were conducted in owls after the completion of visually guided shifts in the representation of ITD. Consequently, we do not know the chronology of changes in ITD tuning in the optic tectum and ICx. Visual instruction 
of the auditory map might occur initially at the level of the optic tectum (or elsewhere) and subsequently be propagated backward through the ITD pathway to the ICx. The optic tectum seems especially plausible as an initial site of visual guidance because of the juxtaposition of auditory and visual maps: vision provides precise spatial information to bimodal cells that participate in the representation of auditory space. Reciprocal connections from the optic tectum to the ICx, which would be required to support such an hypothesis, have been demonstrated for some mammalian species (Benevento and Fallon, 1975; Adams, 1980; Coleman and Clerici, 1987). However, such connections were not observed in a retrograde tracing study in barn owls that examined inputs to the ICx (Knudsen, 1983).

An instructive signal to the ICx may be present only transiently during development. Large visually guided changes in sound localization behavior occur only during restricted early periods of development (Knudsen and Knudsen, 1990). Similar restrictions in the plasticity of the neural representation of auditory space (Knudsen, 1985) might reflect the loss of instructive inputs to the ICx over the course of development. In this context, it is worth noting that anatomical studies of projections to the $\mathrm{ICX}$ were all conducted in adult barn owls, and would have missed developmentally transient inputs.

\section{Comparison with monaural occlusion}

Like displacing prisms, monaural occlusion (plugging one ear) disrupts the normal correspondence between sound localization cues and the apparent locations of sound sources. While prisms achieve this by shifting the visual field and leaving sound localization cues essentially unchanged, monaural occlusion leaves the visual field normal, but attenuates and delays the sounds reaching the occluded ear (Knudsen et al., 1984). Like prism rearing, chronic monaural occlusion in baby owls leads to adaptive shifts in the neural representation of auditory space in the optic tectum: in adult barn owls reared with one ear occluded, the auditory and visual maps of space are mutually aligned as long as the ear remains occluded (despite the abnormal localization cues reaching the eardrum), but are misregistered when normal localization cues are restored (Knudsen, 1985; Mogdans and Knudsen, 1992a):

The mechanisms underlying this plasticity are probably not the same as for prism rearing. Recent experiments have demonstrated that adaptive changes in the neural representation of auditory space can occur in monaurally occluded barn owls reared without vision (Knudsen and Mogdans, 1992). In mammals, monaural occlusion induces changes in binaural properties of neurons at least as early in the ascending auditory pathway as the ICc (Silverman and Clopton, 1977; Moore and Irvine, 1981), and in barn owls plasticity in tuning for ITD occurs at or before the level of the ICx (Mogdans and Knudsen, 1990). Moreover, plasticity in the representation of ILD occurs before the level of the inferior colliculus, at the initial site of binaural comparison (Mogdans and Knudsen, 1992b). Thus, the site and mechanisms of plasticity that underlie the calibration of the auditory space map may vary with the specific nature of the animal's sensory experience.

\section{References}

Adams JC (1980) Crossed and descending projections to the inferior colliculus. Neurosci Lett 19:1-5.

Benevento LA, Fallon JH (1975) The ascending projections of the superior colliculus in the rhesus monkey (Macaca mulatta). J Comp Neurol 160:339-362.

Brainard MS, Knudsen EI, Esterly SD (1992) Neural derivation of sound source location: resolution of spatial ambiguitics in binaural cues. J Acoust Soc Am 91:1015-1027.

Brown TH, Kairiss EW, Keenan CL (1990) Hebbian synapses: biophysical mechanisms and algorithms. Annu Rev Neurosci 13:475511.

Carpenter MB (1959) Lesions of the fastigial nucleus in the rhesus monkey. Am J Anat 104:1-34.

Carpenter MB, Brittin GM, Pines J (1958) Isolated lesions of the fastigial nuclei in the cat. J Comp Neurol 109:65-89.

Carr CE, Konishi M (1988) Axonal delay lines for time measurement in the owl's brainstem. Proc Natl Acad Sci USA 85:8311-8315.

Carr CE, Konishi M (1990) A circuit for detection of interaural time differences in the brain stem of the barn owl. J Neurosci 10:32273246.

Coleman JR, Clerici WJ (1987) Sources of projections to subdivisions of the inferior colliculus in the rat. J Comp Neurol 262:215-226.

Earle AM, Matzke HA (1974) Efferent fibers of the deep cerebellar nuclei in hedgehogs. $J$ Comp Neurol 154:117-132.

Fisher GH (1964) Spatial localization by the blind. Am J Psychol 77: $2-13$.

Fregnac Y, Shulz D, Thorpe S, Bienenstock E (1988) A cellular analogue of visual cortical plasticity. Nature 333:367-370.

Fregnac Y, Shulz D, Thorpe S, Bienenstock E (1992) Cellular analogs of visual cortical epigenesis. I. Plasticity of orientation selectivity. J Neurosci 12:1280-1300.

Fujita I, Konishi M (1991) The role of GABAergic processing of interaural time difference in the owl's auditory system. J Neurosci 11:722-739.

Gaze RM, Keating MJ, Szekeley G, Beazeley L (1970) Binocular interaction in the formation of specific intertectal neuronal connections. Proc R Soc London [Biol] 175:107-147.

Gilbert CD, Wiesel TN (1992) Receptive field dynamics in adult primary visual cortex. Nature 356:150-152.

Gruel JM, Luhmann HJ, Singer W (1988) Pharmacological induction of use-dependent receptive field modifications in the visual cortex. Science 242:74-77.

Hehb DO (1949) The organization of behavior. New York: Wiley.

Heinen SJ, Skavenski AA (1991) Recovery of visual responses in foveal V1 neurons following bilateral foveal lesions in adult monkey. Exp Brain Res 83:670-674.

Hubel DH, Wiesel TN, LeVay S (1977) Plasticity of ocular dominance columns in monkey striate cortex. Philos Trans R Soc Lond [Biol] 278:377-409.

Huffman RF, Henson OJ (1990) The descending auditory pathway and acousticomotor systems: connections with the inferior colliculus. Brain Res Rev 15:295-323.

Itaya SK, Hoesen GW (1982) Retinal innervation of the inferior colliculus in rat and monkey. Brain Res 233:45-52.

Jen LS, So KF, Woo HH (1984) An anterograde HRP study of the retinocollicular pathways in normal hamsters and hamsters with one eye enucleated at birth. Brain Res 294:169-173.

Jenkins WM, Merzenich MM, Ochs MT, Allard T, Guic RE (1990) Functional reorganization of primary somatosensory cortex in adult owl monkeys after behaviorally controlled tactile stimulation. J Neurophysiol 63:82-104.

Jones B (1975) Spatial perception in the blind. Br J Psychol 66:461472.

Kaas JH, Krubitzer LA, Chino YM, Langston AL, Polley EH, Blair N (1990) Reorganization of retinotopic cortical maps in adult mammals after lesions of the retina. Science 248:229-231.

Knudsen EI (1982) Auditory and visual maps of space in the optic tectum of the owl. J Neurosci 2:1177-1194.

Knudsen EI (1983) Subdivisions of the inferior colliculus in the barn owl (Tyto alba). J Comp Neurol 218:174-186.

Knudsen EI (1984) Synthesis of a neural map of space in the owl. In: Dynamic aspects of neocortical function (Edelman GM, Cowan WM, Gall WE, eds), pp 375-396. New York: Wiley.

Knudsen EI (1985) Experience alters spatial tuning of auditory units in the optic tectum during a sensitive period in the barn owl. J Neurosci 5:3094-3109.

Knudsen EI, Brainard MS (1991) Visual instruction of the neural map of auditory space in the developing optic tectum. Science 253:85-87. 
Knudsen EI, Knudsen PF (1983) Space-mapped auditory projections from the inferior colliculus to the optic tectum in the barn owl (Tyto alba). J Comp Neurol 218:187-196.

Knudsen EI, Knudsen PF (1989) Vision calibrates sound localization in developing barn owls. J Neurosci 9:3306-3313.

Knudsen EI, Knudsen PF (1990) Sensitive and critical periods for visual calibration of sound localization by barn owls. J. Neurosci 10: 222-232.

Knudsen EI, Knudsen PF (1993) Parallel pathways mediating both sound localization and gaze control in the forebrain and midbrain of the barn owl. J Neurosci 13:2837-2852.

Knudsen EI, Konishi M (1978) Space and frequency are represented separately in auditory midbrain of the owl. J Neurophysiol 41:870884.

Knudsen EI, Mogdans J (1992) Vision-independent adjustment of unit tuning to sound localization cues in response to monaural occlusion in developing owl optic tectum. J Neurosci 12:3485-3493.

Knudsen EI, Konishi M, Pettigrew JD (1977) Keceptive fields of auditory neurons in the owl. Science 198:1278-1280.

Knudsen EI, Esterly SD, Knudsen PF (1984) Monaural occlusion alters sound localization during a sensitive period in the barn owl. J Neurosci 4:1001-1011.

Knudsen FI, Esterly SD, du Lac S (1991) Stretched and upside-down maps of auditory space in the optic tectum of blind-reared owls: acoustic basis and behavioral correlates. J Neurosci 11:1727-1747.

Konishi M, Takahashi TT, Wagner H, Sullivan WE, Carr CE (1988) Neurophysiological and anatomical substrates of sound localization in the owl. In: Auditory function: neurobiological bases of hearing (Edelman GM, Gall WE, Cowan WM, eds), pp 721-745. New York: Wiley.

Kunzle H (1988) Retinofugal projections in hedgehog-tenrecs (Echinops telfairi and Setifer setosus). Anat Embryol (Berl) 178:77-93.

Lackner JR (1973) Visual rearrangement affects auditory localization. Neuropsychol 11:29-32.

LeVay S, Wiesel TN, Hubel DH (1980) The development of ocular dominance columns in normal and visually deprived monkeys. $\mathrm{J}$ Comp Neurol 191:1-51.

Lisberger SG (1988) The neural basis for learning of simple motor skills. Science 242:728-735.

Merzenich M, Nelson R, Stryker M, Cynader M, Schoppmann A, Zook J (1984) Somatosensory cortical map changes following digit amputation in adult monkeys. J Comp Neurol 224:591-605.

Mogdans J, Knudsen EI (1990) Auditory experience modifies the tuning for sound localization cues of neurons in the barn owl's optic tectum and inferior colliculus. Soc Neurosci Abstr 16:828.

Mogdans J, Knudsen EI (1992a) Adaptive adjustment of unit tuning to sound localization cues in response to monaural occlusion in developing owl optic tectum. J Neurosci 12:3473-3484.

Mogdans J, Knudsen EI (1992b) A site of plasticity in the brainstem of the barn owl: regulation of inhibition in response to early monaural occlusion. Soc Neurosci Abstr 18:383.

Moiseff A (1989) Binaural disparity cues available to the barn owl for sound localization. J Comp Physiol [A] 164:629-636.

Moiseff A, Konishi M (1981a) Neuronal and bchavioral sensitivity to binaural time differences in the owl. J Neurosci 1:40-48.
Moiseff A, Konishi M (1981b) The owl's interaural pathway is not involved in sound localization. J Comp Physiol 144:299-304.

Moore DR, Irvine DR (1981) Plasticity of binaural interaction in the cat inferior colliculus. Brain Res 208:198-202.

Norris JC (1982) Sensitivity to sound location and other response properties of auditory neurons in the cerebellum of the owl. $\mathrm{PhD}$ thesis, Stanford University.

Olsen JF, Knudsen EI, Esterly SD (1989) Neural maps of interaural time and intensity differences in the optic tectum of the barn owl. J Neurosci 9:2591-2605.

Robertson D, Irvine DR (1989) Plasticity of frequency organization in auditory cortex of guinca pigs with partial unilateral deafness. $\mathbf{J}$ Comp Neurol 282:456-471.

Shatz CJ, Stryker MP (1978) Ocular dominance in layer IV of cat's visual cortex and the effects of monocular deprivation. J Physiol (Lond) 281:267-283.

Shulz D, Fregnac Y (1992) Cellular analogs of visual cortical epigenesis. II. Plasticity of binocular integration. J Neurosci 12:1301-1318.

Silverman MS, Clopton BM (1977) Plasticity of binaural interaction. I. Effect of early auditory deprivation. J Neurophysiol 40:1266-1274.

Spiegelman MN, Bryden MP (1967) Effects of early and late blindness on auditory spatial learning in the rat. Neuropsychologia 5:267-274.

Sullivan WE, Konishi M (1986) A neural map of interaural phase difference in the owl's brainstem. Proc Natl Acad Sci USA 83:84008404.

Takahashi T, Konishi M (1986) Selectivity for interaural time difference in the owl's midbrain. J Neurosci 6:3413-3422.

Takahashi T, Konishi M (1988) Projections of the cochlear nuclei and nucleus laminaris to the inferior colliculus of the barn owl. J Comp Neurol 274:190-211.

Takahashi T, Carr CE, Brecha N, Konishi M (1987) Calcium binding protein-like immunoreactivity labels the terminal field of nucleus laminaris of the barn owl. J Neurosci 7:1843-1856.

Takahashi T, Wagner H, Konishi M (1989) Role of commissural projections in the representation of bilateral auditory space in the barn owl's inferior colliculus. J Comp Neurol 281:545-554.

Udin SB, Fawcett JW (1988) Formation of topographic maps. Annu Rev Neurosci 11:289-327.

Udin SB, Keating MJ (1981) Plasticity in a central nervous pathway in Xenopus: anatomical changes in the isthmotectal projection after larval eye rotation. J Comp Neurol 203:575-594.

Wagner H, Takahashi T, Konishi M (1987) Representation of interaural time difference in the central nucleus of the barn owl's inferior colliculus. J Neurosci 7:3105-3116.

Wanet MC, Veraart C (1985) Processing of auditory information by the blind in spatial localization tasks. Percept Psychophys 38:91-96.

Yamauchi K, Yamadori T (1982) Retinal projection to the inferior colliculus in the rat. Acta Anat (Basel) 114:355-360.

Zimminy R, Sobusiak T, Deja A, Kotecki A (1981) Cerebellar afferent projection to the inferior colliculus of the mesencephalic tectum. An anterograde degeneration study in the rabbit. Z Mikrosk-Anat Forsch 95:197-209. 